\title{
Gut metagenomics discriminates unique microbial signatures in diverse symptomatic profiles with attention-deficit/hyperactivity disorder
}

Yan Li

Xi'an Jiaotong University Medical College First Affiliated Hospital https://orcid.org/0000-0002-74657705

Haiting Sun

the Fourth Military Medical University

Yufen Huang

BGI Genomics

Anqi Yin

the Fourth Military Medical University

Ping Wang

the Fourth Military Medical University

Jiao Han

Xi'an Jiaotong University

Dingding Qu

Xi'an Jiaotong University

Lixia Zhuo

Xi'an Jiaotong University

Mei Tian

the Fourth Military Medical University

Chen Wang

the Fourth Military Medical University

Fangfang Jian

the Fourth Military Medical University

Jinyang Zhao

BGI Genomics

Silong Sun

BGI Genomics

\section{Luis Augusto Rohde}

Universidade Federal do Rio Grande do Sul Instituto de Filosofia e Ciencias Humanas

\section{Xuefeng Xie}

BGI Genomics 


\section{Xin Sun ( $\square$ sunxin6@fmmu.edu.cn )}

the Fourth Medical University

\section{Lize Xiong}

the Fourth Military Medical University

\section{Research}

Keywords: ADHD, Gut microbiota, Microbiota-gut-brain axis, Metagenomic sequencing, Biomarker

Posted Date: May 15th, 2020

DOI: https://doi.org/10.21203/rs.3.rs-28862/v1

License: (c) (1) This work is licensed under a Creative Commons Attribution 4.0 International License.

Read Full License 


\section{Abstract}

\section{Background}

Attention-deficit/hyperactivity disorder (ADHD) is a highly heterogeneous psychiatric disorder that can be divided into inattentive (I-ADHD), hyperactive-impulsive (HI-ADHD), and combined (C-ADHD) subtypes. Different early life events and environmental factors correlated with the gut microbiota community have been implicated in the development of ADHD. However, whether different ADHD symptomatic presentations are associated with distinct microbiota composition and function still unknown. Therefore, we carried out metagenomic analysis from 207 subjects to characterize the gut microbial profiles in ADHD and subgroup patients.

Results

The current study revealed that the gut microbiota composition (beta diversity) can be effectively distinguished between C-ADHD patients and HCs, but not I-ADHD patients and HCs, nor general ADHD patients and HCs. Features include underrepresentation of 8 species belonging to the genus Bacteroides and enrichment of 5 species of Bifidobacterium and Prevotella in general ADHD patients (all $p<0.05$ ). Eight of the above species became progressively reduced (ovatus, thetaiotaomicron, intestinalis, cellulosilyticus, and fluxus belonging to the genus Bacteroides) or enriched (Prevotella_copri, Prevotella_buccae and Bifidobacterium_breve) from healthy controls (HCs) to I-ADHD and C-ADHD patients. Predicted metabolic functions from these distinguished gut microbial markers described a certain compensatory host metabolism in ADHD and subgroup patients. Particularly, pyridoxal 5'phosphate (a dominant vitamin B6 active type) biosynthesis pathways were significantly reduced in CADHD patients, because serum vitamin B6 deficiency in ADHD patients was found previously. Of note, we identified diverse virulence factor and antibiotic resistance from the gut microbiota of ADHD patients. The abundance of antibiotic resistance ontology ANT(9)-la positively correlated with the abundance of Prevotella_amnii, which was enriched in ADHD patients. Moreover, species-based bacterial markers were used to construct classifiers and achieved a higher AUC of 0.87 in C-ADHD vs. HC than that in ADHD vs. HC (AUC = 0.84).

Conclusions

These findings uncover alterations in microbial composition in subgroup patients and provide potential biomarkers for diagnosis different symptomatic presentations for ADHD.

Trial registration: ClinicalTrials.gov, NCT03447223. Registered 27 February 2018, https://clinicaltrials.gov/ct2/show/NCT03447223?term=03447223\&draw=2\&rank=1

\section{Background}


Attention deficit/hyperactivity disorder (ADHD), a childhood-onset psychiatric disorder, has a worldwide prevalence of approximately $5 \%[1,2]$. According to its syndromic profiles, this neurodevelopmental disorder can be divided into inattentive (I-ADHD), hyperactive-impulsive (HI-ADHD), and combined (C$A D H D)$ presentations $[1,3]$. ADHD is associated with highly heterogeneous impairment in cognitive and social functions and may result in future poor lifetime outcomes such as academic failure and mental illness $[1,4]$. It is important to highlight that the different ADHD symptomatic profiles are associated with diverse types and levels of negative outcomes [3]. Although gene-environment interactions are implicated in ADHD development $[5,6]$, the underlying pathophysiological mechanisms remain largely unknown.

Recent studies have provided growing evidences that gut microbiota dysbiosis in childhood or adulthood may increase the risk of psychiatric disorders, such as major depressive disorder [7], bipolar disorder [8], schizophrenia [9], autism spectrum disorder [10], and ADHD [11]. These results suggest a role of the gut microbiota in brain function and behavior and support communication between the gut and the brain (microbiota-gut-brain axis) [12,13]. Of note, many environmental risk factors of ADHD development, such as cesarean delivery [14], formula feeding [15], antibiotic use [16], and diet style[17] are also associated with gut bacterial compositions.

Studies based on 16S rRNA sequencing have preliminarily distinguished different gut microbiota between ADHD patients and healthy controls (HCs) from several small size cohorts [18-22]. However, no studies have been carried out to compare bacterial variation between patients with different ADHD symptomatic profiles and HCs. Since there are highly heterogeneous neurobehavioral deficits among patients with different ADHD symptomatic profiles, we might lose relevant specificities when trying to distinguish the bacterial taxa between a mixed and heterogeneous group of ADHD patients and HCs. In addition, the 16S rRNA sequencing approach may omit some key information owing to the limited taxonomic and functional resolution level.

Therefore, in this study, metagenomics sequencing was performed to discriminate microbial composition and function between subgroups of patients with ADHD. The identified gut microbial biomarkers require further evaluation for its diagnostic performance for classifying different symptomatic profiles of patients with ADHD.

\section{Results}

\section{Demographic and clinical characteristics of the recruited participants}

A total of 207 children and adolescents were recruited, including 98 ADHD patients (38 I-ADHD, 53 CADHD, $7 \mathrm{HI}-A D H D$ ) and $109 \mathrm{HCs}$. As the number of subjects with HI-ADHD was too low to compare with other subgroups and HC, ultimately, 38 I-ADHD, 53 C-ADHD and 109 HC were included in the subgroup analysis. The general demographic characteristics from the recruited subjects are displayed in Table 1 (I- 
ADHD, C-ADHD and HC), Table S1 (Supporting Information, ADHD and HC), and Table S11 (Supporting Information). There were no significant differences among the three groups in age, body mass index (BMI), premature birth, maternal pregnancy with metabolic disease, or antibiotic use during pregnancy or infancy. The proportion of male subjects among C-ADHD patients was higher than that among HCs (98.1\% vs $81.7 \%, p=0.007$ ). The proportion of only children among C-ADHD patients was lower than that among HCs $(39.6 \%$ vs $65.1 \%, p=0.007)$. The percent of cesarean deliveries among I-ADHD patients was lower than that among $\mathrm{HCs}(36.8 \%$ vs $60.6 \%, p=0.023)$. Furthermore, low birth weight occurred more often in C-ADHD patients than in HCs $(20.8 \%$ vs $5.5 \%, p=0.012)$. Patients in I-ADHD and C-ADHD had lower scores on the intelligence quotient (IQ) $(p<0.001)$. Predictably, all ADHD diagnostic indices, including the Diagnostic and Statistical Manual of Mental Disorders_Attention Deficits (DSM_AD) scores, DSM_Hyperactivity/Impulsivity Deficits (DSM_HD) scores, and total Conners Parent Rating Scales (CPRS) scores, were higher in I-ADHD and C-ADHD patients. 
Table 1

The baseline characteristics of ADHD subgroup patients $(I-A D H D=38 ; C-A D H D=53)$ and HCs $(n=109)$ in the study cohort.

\begin{tabular}{|c|c|c|c|c|}
\hline Characteristics & $\begin{array}{l}\text { I-ADHD } \\
(n=38)\end{array}$ & $\begin{array}{l}\text { C-ADHD } \\
(n=53)\end{array}$ & $\begin{array}{l}\mathrm{HC} \\
(n=109)\end{array}$ & ${ }^{*} p$ value \\
\hline Age, years, mean \pm S.D. & $9.4 \pm 2.1$ & $8.8 \pm 1.9$ & $8.9 \pm 1.8$ & 0.372 \\
\hline $\mathrm{BMI}, \mathrm{kg} / \mathrm{m}^{2}$, mean \pm S.D. & $17.3 \pm 3.9$ & $16.9 \pm 2.7$ & $17.1 \pm 4.3$ & 0.737 \\
\hline Male, No. (\%) & $33(86.8)$ & $52(98.1)$ & $89(81.7)$ & $0.007^{b}$ \\
\hline IQ, mean \pm S.D. & $\begin{array}{l}103.0 \pm \\
14.5\end{array}$ & $\begin{array}{l}101.9 \pm \\
13.5\end{array}$ & $\begin{array}{l}112.0 \pm \\
13.5\end{array}$ & $\begin{array}{l}4.399 \mathrm{E}- \\
05^{\mathrm{ab}}\end{array}$ \\
\hline Only child, No. (\%) & $19(50.0)$ & $21(39.6)$ & $71(65.1)$ & $0.007^{b}$ \\
\hline $\begin{array}{l}\text { Maternal pregnancy with metabolic disease, } \\
\text { No. }(\%)\end{array}$ & $0(0)$ & $2(3.8)$ & $2(1.8)$ & 0.645 \\
\hline Cesarean section, №. (\%) & $14(36.8)$ & $24(45.3)$ & $66(60.6)$ & $0.023^{\mathrm{a}}$ \\
\hline Premature birth, < 37 weeks, No. (\%) & $2(5.3)$ & $4(7.5)$ & $6(5.5)$ & 0.920 \\
\hline Low birth weight, < 2.5 kg, No. (\%) & $5(13.2)$ & $11(20.8)$ & $6(5.5)$ & $0.012^{b}$ \\
\hline $\begin{array}{l}\text { Maternal antibiotic use during pregnancy, No. } \\
(\%)\end{array}$ & $2(5.3)$ & $0(0)$ & $1(0.9)$ & 0.151 \\
\hline Antibiotic use during infancy, No. (\%) & $5(13.2)$ & $4(7.5)$ & $8(7.3)$ & 0.510 \\
\hline DSM_AD scores, mean \pm S.D. & $6.6 \pm 0.9$ & $7.5 \pm 1.2$ & $1.6 \pm 1.1$ & $\begin{array}{l}4.728 \mathrm{E}- \\
34^{\mathrm{abc}}\end{array}$ \\
\hline DSM_HD scores, mean \pm S.D. & $1.9 \pm 1.1$ & $7.0 \pm 1.6$ & $0.9 \pm 0.9$ & $\begin{array}{l}2.010 \mathrm{E}- \\
29^{\mathrm{abc}}\end{array}$ \\
\hline Total CPRS scores & $4.2 \pm 1.7$ & $6.4 \pm 2.5$ & $1.7 \pm 1.1$ & $\begin{array}{l}6.902 \mathrm{E}- \\
28^{\mathrm{abc}}\end{array}$ \\
\hline
\end{tabular}

S.D., standard deviation; $\mathrm{BMI}$, body mass index; IQ, intelligence quotient; DSM, the diagnostic and statistical manual of mental disorders; DSM_AD, DSM attention deficits; DSM_HD, DSM hyperactivity/impulsivity deficits; CPRS, Conners' Parent Rating Scales. * $p$ value based on the KruskalWallis test (continuous variables, or the Wilcoxon rank-sum test for two groups) or Fisher's exact test (categorical variables) for all groups. ${ }^{\mathrm{a}} p<0.05$ for I-ADHD and HC; ${ }^{\mathrm{b}} p<0.05$ for C-ADHD and HC; ${ }^{\mathrm{c}} p<$ 0.05 for I-ADHD and C-ADHD. 


\begin{tabular}{|c|c|c|c|c|}
\hline Characteristics & $\begin{array}{l}\text { I-ADHD } \\
(n=38)\end{array}$ & $\begin{array}{l}\text { C-ADHD } \\
(n=53)\end{array}$ & $\begin{array}{l}\mathrm{HC} \\
(n=109)\end{array}$ & ${ }^{*} p$ value \\
\hline Rutter types, №. (\%) & $9(23.7)$ & $34(64.2)$ & $2(1.8)$ & \multirow{4}{*}{$\begin{array}{l}1.464 \mathrm{E}- \\
06^{\mathrm{bc}}\end{array}$} \\
\hline A & $12(31.6)$ & $3(5.7)$ & $10(9.2)$ & \\
\hline $\mathrm{N}$ & $5(13.2)$ & 7 (13.2) & $4(3.7)$ & \\
\hline \multicolumn{4}{|l|}{ M } & \\
\hline \multicolumn{5}{|c|}{$\begin{array}{l}\text { S.D., standard deviation; BMI, body mass index; IQ, intelligence quotient; DSM, the diagnostic and } \\
\text { statistical manual of mental disorders; DSM_AD, DSM attention deficits; DSM_HD, DSM } \\
\text { hyperactivity/impulsivity deficits; CPRS, Conners' Parent Rating Scales. }{ }^{*} p \text { value based on the Kruskal- } \\
\text { Wallis test (continuous variables, or the Wilcoxon rank-sum test for two groups) or Fisher's exact test } \\
\text { (categorical variables) for all groups. }{ }^{a} p<0.05 \text { for I-ADHD and HC; }{ }^{b} p<0.05 \text { for C-ADHD and HC; }{ }^{c} p< \\
0.05 \text { for I-ADHD and C-ADHD. }\end{array}$} \\
\hline
\end{tabular}

The dietary and defecation habits among I-ADHD, C-ADHD and HC are listed in Table S2 and Table S3 (Supporting Information). We found no significant differences among the three groups in all dietary and defecation habits, including infant feeding, the preference of side dishes, staple foods, yogurt and other fermented food, and defecation frequency, smoothing, and shape.

\section{Fecal microbiome diversity in ADHD and subgroup patients}

The alpha diversity of gut microbiota was estimated between ADHD or subgroup patients and HCs. We found significantly lower gene numbers in ADHD patients than in $\mathrm{HCs}(p=0.042)$, though the gut microbiota richness was similar between the two groups (Shannon index, $p=0.076$, Fig. $1 \mathrm{a}$ ). Subgroup gene numbers demonstrated that there was lower dissimilarity between I-ADHD and HC $(p=0.11)$ than between C-ADHD and HC ( $p=0.017$, Fig. $1 \mathrm{~b})$. However, alpha diversity analysis revealed no significant difference between any two groups among C-ADHD, I-ADHD and HC.

To assess whether the gut microbiota can be effectively distinguished between the groups, supervised analysis with spare PLS-DA (sPLS-DA) and permutational multivariate analysis of variance (PERMANOVA) were performed at the gene family level. From the sPLS-DA results, samples of ADHD patients and HCs were clustered into two groups, though the PERMANOVA results suggested that the microbial composition was not significantly different between the two groups ( $p=0.227$, Fig. $1 c)$. However, there was a significant dissimilarity between C-ADHD and HC ( $p=0.020$, Fig. 1d). No significant findings were obtained between I-ADHD vs. $\mathrm{HC}(p=0.134)$ and I-ADHD vs. C-ADHD $(p=0.519)$. Together, these results suggest greater gut microbiota variation between C-ADHD and HC than between ADHD and $\mathrm{HC}$.

PERMANOVA was performed to explore the influence of other host characteristics on gut microbiota. In addition to the ADHD subgroups, sex and IQ were also significantly associated with gut microbial composition (Table S4, Supporting Information). Of note, there was a higher proportion of male subjects among C-ADHD subgroup than that among HCs. To address whether the gut microbiota differences 
found in Fig. 1 just translate sex difference between two groups, we further performed analyses stratified for males. The gut microbiota richness (Shannon index, $p=0.076$ ) in male subjects between ADHD and HCs was the same as mixed sex analysis. The gene numbers $(p=0.057)$ and gut microbiota richness (Shannon index, $p=0.076$ ) was similar in male subjects between ADHD patients and HCs (Figure S1a). The gene numbers $(p=0.017)$ and gut microbiota richness (Shannon index, $p=0.076)$ in male subjects between C-ADHD and HC were similar to mixed sex analyses (Figure S1b). PERMANOVA results showed a more significant difference in male samples between C-ADHD and $\mathrm{HC}(p<0.001$, Figure $\mathrm{S} 1 \mathrm{~d})$, compared with ADHD and HC ( $p=0.028$, Figure S1c). Therefore, unmatched sex here didn't alter the outcome that greater gut microbiota variation between C-ADHD and HC than between ADHD and HC.

\section{ADHD and subgroups are associated with distinct microbial compositions}

Metagenomic sequencing analysis (linear discriminant analysis effect size (LEfSe)) identified 12 bacterial taxa enriched in ADHD patients and 33 enriched in HCs (Fig. 2a). At the bacterial phylum level, Bacteroidetes, Firmicutes, Proteobacteria and Actinobacteria were the most abundant taxa in both groups (Table S5, Supporting Information). The HCs displayed a higher abundance of Fusobacteria than the ADHD patients. At the order and family levels, the relative abundances of members of the orders Fusobacteriales, Flavobacteriales, Rhodocyclales and Sphigomonadales and of the families Fusobacteriaceae, Flavobacteriaceae, Rhodocyclaceae, Sphigomonadaceae and Bacillales_noname were lower in the ADHD patients than in the HCs, whereas the relative abundance of members of the family Prevotellaceae was higher in the ADHD patients. At the species level, eight of the top $18 \mathrm{HC}$-enriched species (ovatus, fragilis, thetaiotaomicron, intestinalis, cellulosilyticus, salyersiae, fluxus, and nordii) belonged to the genus Bacteroides. Species in Bifidobacterium (breve and bifidum) and Prevotella (amnii, buccae and copri) were more abundant in the ADHD patients than in the HCs. A cladogram of significantly different taxa is shown in Fig. $2 \mathrm{~b}$, and an overview of the relative abundance of different bacteria at the genus level between ADHD patients and HCs is shown in Table S6 (Supporting Information).

We next compared the gut microbial compositions in subgroups of patients with ADHD and HCs. Detailed information on the significantly different bacterial taxa among I-ADHD, C-ADHD and HC is shown in Table S7 and Table S8 in the Supporting Information. We compared the numbers of significantly different bacterial taxa among the groups, which were, from more to less in proper order, C-ADHD vs. HC (70), IADHD vs. C-ADHD (34), and I-ADHD vs. HC (24) (Fig. 3a). Moreover, we found that the bacterial difference patterns in C-ADHD vs. HC and C-ADHD vs. I-ADHD were largely shared (Fig. 3a, b). Thirteen gut bacterial taxa abundant in HC compared with C-ADHD were also enriched in I-ADHD compared with C-ADHD. These shared abundances in I-ADHD and HC were Rhizobiales, Oscillospiraceae, Bilophila, Oscillibacter, Subdoligranulum, Bacteroides_cellulosilyticus, Bacteroides_fluxus, Bacteroides_nordii, Bacteroides_ovatus, Lachnospiraceae_bacterium, Bilophila_wadsworthia, Oscillibacter_unclassified, and Subdoligranulum_unclassified. The other 5 bacterial taxa (Listeriaceae, Prevotellaceae, Veillonellaceae, Listeria, Listeria_marthii) were highly enriched in C-ADHD compared with I-ADHD or HC. The specific 
different bacterial taxa between I-ADHD vs. HC, C-ADHD vs. HC and I-ADHD vs. C-ADHD are shown in Figure S2 (Supporting Information). Together, these data provided initiatory evidence of greater dissimilarity in gut microbial composition between C-ADHD vs. HC than between I-ADHD vs. HC. Notably, C-ADHD patients can be distinguished from I-ADHD patients by different gut microbiota profiles.

In addition, the Jonckheere-Terpstra test identified a progressive scarcity or prevalence from HCs to IADHD and C-ADHD patients (Fig. 3c). We found that Prevotella_copri, Prevotella_buccae and Bifidobacterium_breve were progressively enriched from HC to I-ADHD and C-ADHD, while progressively reduced enrichment of the species ovatus, thetaiotaomicron, intestinalis, cellulosilyticus, and fluxus belonging to the genus Bacteroides were also identified. Therefore, these results suggest that the progressively increased or reduced enrichment of microbial taxa may be associated with the severity of ADHD development.

\section{Distinct bacterial functional profiles in ADHD and subgroups}

Microbial metagenomic sequencing data were used to predict discrepancy in functional metabolic pathways in ADHD and subgroup patients. Altogether, 362 pathways from the MetaCyc metabolic pathway database that presented in more than $10 \%$ of samples were identified and analyzed. A total of 9 pathways were significantly different between ADHD patients and HCs ( $p<0.05$, LDA score > 2; Fig. 4a). LEfSe revealed that only bacterial xylose degradation IV was significantly enriched in the ADHD patients, while bacterial pathways for inosine-5'-phosphate biosynthesis II, flavin biosynthesis III (fungi), Lphenylalanine degradation IV, adenine and adenosine salvage III, starch degradation V, hydrogen production VIII, purine ribonucleoside degradation and L-rhamnose degradation I were significantly reduced in the ADHD patients.

Subsequently, the diversity of functional pathways in ADHD subgroups was identified. The C-ADHD subgroup had predicted enrichment in bacterial pathways for S-adenosyl-L-methionine cycle I and xylose degradation and reduction in GDP-mannose-derived 0-antigen building blocks biosynthesis, pantothenate and coenzyme A biosynthesis III, L-histidine biosynthesis, L-arginine biosynthesis III, L-rhamnose degradation I, flavin biosynthesis III, inosine-5'-phosphate biosynthesis I, pyridoxal 5'-phosphate biosynthesis and salvage and NAD salvage pathway I, compared to HC (Fig. 4b). In the I-ADHD subgroup, the predicted bacterial pathways of L-lysine, L-threonine and L-methionine biosynthesis I, anhydromuropeptide recycling, tetrahydrofolate biosynthesis and salvage and heme biosynthesis from glycine were increased, whereas pyrimidine ribonucleotide de novo biosynthesis, purine ribonucleoside degradation, GDP-mannose biosynthesis and stachyose degradation were decreased compared to those in the HC. Of note, the Venn diagram showed zero overlap in diverse pathway profiles in C-ADHD vs. HC and I-ADHD vs. HC (Fig. 4c), suggesting distinct gut bacterial functions in ADHD subgroups. The specific different bacterial functional pathways between I-ADHD vs. HC, C-ADHD vs. HC and I-ADHD vs. C-ADHD are shown in Figure S3 (Supporting Information).

The result of association analysis between metabolic pathways and gut microbial composition demonstrated that there were 14 significantly different species associated with metabolic pathways. We 
found that Prevotella_copri, which was enriched in C-ADHD, was positively associated with enrichment in the bacterial S-adenosyl-L-methionine cycle I pathway compared with HC. There was an overrepresentation of Bifidobacterium_longum, Bifidobacterium_pseudocatenulatum and Bifidobacterium_catenulatum in I-ADHD, which was positively correlated with the increased superpathway of L-lysine, L-threonine and L-methionine biosynthesis I and the superpathway of pyrimidine ribonucleoside degradation compared with C-ADHD or HC. Bacteroides_ovatus, Bacteroides_nordii, Lachnospiraceae_bacterium_5_1_57FAA and Lachnospiraceae_bacterium_7_15_8FAA enriched in both IADHD and $\mathrm{HC}$ were positively associated with enrichment of the superpathway of GDP-mannose-derived 0 -antigen building blocks biosynthesis in these patients (Fig. 5). These findings suggest that there was a certain agreement in the variation tendency between intestinal species abundance and its metabolic functions.

\section{ADHD patients show diverse virulence factor and antibiotic resistance in their gut microbiota}

Metagenomic sequencing analysis provides the chance to study the gut bacterial virulence factor and antibiotic resistance in the patients with ADHD on a large size. We did not find significantly differences in the numbers of virulence factor $(p=0.064)$ and antibiotic resistance ontology (ARO) $(p=0.9)$ between ADHD patients and HCs (Figure S4a, Supporting Information). Subgroup virulence factor and ARO numbers demonstrated that there was also no significant difference between any two groups among CADHD, I-ADHD and HC (Figure S4b, Supporting Information). Moreover, we found the increased abundance of 7 and 2 virulence factors in ADHD patients and HCs, respectively (Figure S4c and Table S9, Supporting Information). However, none of these virulence factors was associated with gut microbial taxonomy abundance.

We also identified the overrepresentation of 3 and 4 ARO in ADHD patients and HCs, respectively (Fig. 6a and Table S10, Supporting Information). Interestingly, the abundance of ARO CcrA correlated with the abundance of Bacteroides_ fragilis, which was enriched in HCs. The enrichment of ARO ANT(9)-la correlated with the abundance of Prevotella_amnii, which was overrepresented in ADHD patients (Fig. 6b).

\section{Gut microbial taxa associated with ADHD clinical characteristics}

The associations of gut microbial compositions and clinical symptoms in ADHD were also assessed. Spearman's rank correlation analyses showed significant correlations of bacterial species with symptom severity scores (Fig. 7). The abundant species in C-ADHD compared with HC, Prevotella_buccae, Bifidobacterium_breve, and Bifidobacterium_bifidum were enriched and positively associated with the scores of both total CPRS and DSM. Interestingly, several species belonging to the genus Bacteroides that were abundant in HCs were negatively correlated with DSM_HD and/or DSM_AD scores. We found that increased relative abundances of Bacteroides_nordii, Bacteroides_cellulosilyticus and Bacteroides_intestinalis were associated with fewer symptoms in both hyperactivity/impulsivity 
(DSM_HD scores) and inattention (DSM_AD scores). While Bacteroides_thetaiotaomicron and Bacteroides_ovatus were negatively associated only with DSM_AD scores.

\section{ADHD classification based on gut microbiota profiles}

Random forest (RF) classifications were constructed based on the species level. The relative abundance of 6 bacterial species (Figure S5a, Supporting Information) distinguished the ADHD patients from the HCs (Fig. 8a; AUC = 0.84, 95\% CI 0.78-0.89). For ADHD subgroup classifications, 8 selected species (Figure S5b, Supporting Information) distinguished the C-ADHD patients from HCs (Fig. 8b) with an AUC of 0.87 (95\% $\mathrm{Cl} 0.80-0.93$ ), while the relative abundance of 35 bacterial species (Figure S5c, Supporting Information) distinguished the I-ADHD and C-ADHD subgroups (Fig. 8c; AUC = 0.76, 95\% Cl 0.65-0.86). However, no species was found that could be used as marker to distinguish I-ADHD patients and HCs.

These results suggest that the classifier was able to differentiate ADHD or C-ADHD patients from HCs with good performance. Differentiation effect of the outcome, in turn, was C-ADHD vs. HC, ADHD vs. HC, IADHD vs. C-ADHD, and I-ADHD vs. HC. Therefore, the gut microbial variation in C-ADHD subgroup patients was greater than that in ADHD patients compared with HCs. In addition, the bacterial difference between two ADHD subgroups, C-ADHD and I-ADHD, was striking.

\section{Discussion}

In the present study, we characterized the gut bacterial metagenomic profiles in ADHD as a general group and its two major symptomatic presentations. We also identified several microbial taxa that were associated with the clinical parameters and severity of ADHD. Moreover, we built a bacterial speciesbased classification, which exhibits a higher AUC in C-ADHD versus HC than that in general ADHD patients vs. HCs. These findings not only confirm alterations in the gut microbiome composition of ADHD patients [18-21] but more importantly provide new microbial biomarkers to discriminate subgroups of ADHD.

The metagenomic data here may not directly compare with those results from 16S rRNA sequencing analysis [18-22]. Nevertheless, our findings demonstrated gut dysbiosis in patients with ADHD compared with $\mathrm{HCs}$, which was consistent with previous studies. We found a significantly lower gene number in ADHD or C-ADHD patients than in HCs, though the gut microbiota richness, namely, alpha diversity, was similar between any two groups. There were also no significant differences in alpha diversity between ADHD patients and HCs in the Dutch [18] and Chinese (Zhejiang and Beijing) cohorts [19, 23]. However, two other cohorts from Germany [20] and China (Taiwan) [21], showed reduced or increased alpha diversity in ADHD patients, respectively. Regarding beta diversity, the current study revealed that the gut microbiota can be effectively distinguished between C-ADHD patients and HCs, but not between ADHD patients and HCs. We further found much dissimilarity between C-ADHD patients and HCs than comparing patients with ADHD as whole group and HCs. Of note, these results indicated that the analysis between ADHD subgroups and HC could reveal more significant bacterial diversity, which may be attenuated by comparing total ADHD patients and HCs. 
Notably, the reported distinct bacterial taxa between ADHD patients and HCs were highly inconsistent in previous studies [18-23]. The disturbed bacterial taxa in one cohort may never be replicated or may be opposite in abundance in another cohort. This diversity might reflect the cohort differences in age, sex, region, diet, medication use, early life environment, maternal health, and cesarean delivery, since all these factors could affect gut microbial composition. Here, we found underrepresentation of 8 species (ovatus, fragilis, thetaiotaomicron, intestinalis, cellulosilyticus, salyersiae, fluxus, and nordii) belonging to the genus Bacteroides in ADHD patients. Members of the genus Bacteroides are usually beneficial for gut function and are correlated with neurodevelopment [24, 25]. In addition, species in Bifidobacterium (breve and bifidum) and Prevotella (amnii, buccae and copri) were more abundant in the ADHD patients than that in the HCs. Although we did not find considerable overlap with the previously reported microbial signature of ADHD, the increased Bifidobacterium_breve and Bifidobacterium_bifidum agreed with the results from the Dutch cohort study [18]. The genus Bifidobacterium has been reported to affect the level of enzymes associated with the synthesis of phenylalanine, the precursor of dopamine $[18,26]$. Dopamine, an important monoaminergic neurotransmitter, has been implicated in the pathophysiology of $\operatorname{ADHD}[27,28]$.

In terms of ADHD subgroups, the numbers of distinct bacterial taxa from more to less in proper order were C-ADHD vs. HC, I-ADHD vs. C-ADHD, and I-ADHD vs. HC. The bacterial difference patterns between C$A D H D$ vs. $H C$ and I-ADHD vs. C-ADHD were largely shared. We found a slight bacterial difference between I-ADHD patients and HCs, and we speculate that relatively slight symptoms in I-ADHD may be involved in this difference. Thus, we identified several progressively enriched microbial taxa from HC to I-ADHD and C-ADHD, which is consistent with the clinical severity of $\operatorname{ADHD}[3,29]$. Interestingly, the enrichment of the species Prevotella_copri, Prevotella_buccae and Bifidobacterium_breve progressively increased, while ovatus, thetaiotaomicron, intestinalis, cellulosilyticus and fluxus belonging to the genus Bacteroides progressively decreased from HCs to I-ADHD and C-ADHD patients. Moreover, these species in Prevotella, Bifidobacterium and Bacteroides were also associated with hyperactivity/impulsivity and/or inattention symptoms. Taken together, these results further suggest a more significant dissimilarity in C-ADHD vs. HC compared with ADHD vs. HC. Moreover, there were distinguished gut microbial patterns in the I-ADHD and C-ADHD subgroup patients. We can obtain more accurate gut microbial information from subgroup analysis, which is potentially helpful in the diagnosis of ADHD subtypes.

The functional metabolic pathways predicted from fecal metagenomic analysis exhibited additional divergence in ADHD and subgroup patients. We found that functions correlated with energy regulation in host metabolism, including inosine-5'-phosphate biosynthesis, flavin biosynthesis, adenine and adenosine salvage and purine ribonucleoside degradation, were reduced in ADHD patients. However, it is unclear whether these alterations contribute to abnormal host symptoms, though aberrant brain energy metabolism is involved in some psychiatric disorders [30]. The starch degradation, hydrogen production and rhamnose degradation pathways were also predicted to be decreased in ADHD patients, and these metabolic pathways were associated with host gut functions [31-33]. Moreover, relatively enriched xylose degradation and reduced phenylalanine degradation pathways in ADHD patients are particularly striking. The disturbance of xylose metabolism is implicated in Drosophila hyperactivity behavior [34]. 
Phenylalanine is the precursor of dopamine, which is well studied and known as a dominant neurotransmitter deficit in ADHD pathophysiology. Although very few functional analyses predicted bacterial profiles in previous studies, the increased levels of predicted cyclohexadienyl dehydratase, responsible for phenylalanine synthesis, from the study of the Dutch cohort [18] and the current data together suggest a critical role of abnormal phenylalanine metabolism in ADHD patients. We hypothesized that insufficient dopamine signals in the brain induce potentially compensated precursor production through the gut microbiota.

The functional analysis in subgroups showed some shared and distinct differential metabolic pathways in C-ADHD patients compared with ADHD patients. Notably, arginine and pyridoxal 5'-phosphate biosynthesis pathways were specifically reduced in C-ADHD patients. Arginine, a precursor of nitric oxide, is related to better memory [35] and improved intestinal inflammation [36]. Pyridoxal 5'-phosphate is a dominant vitamin B6 active type, and serum vitamin B6 was decreased in ADHD patients [37]. Moreover, there was no overlap in diverse pathways between C-ADHD vs. HC and I-ADHD vs. HC, suggesting distinct gut bacterial functions in ADHD subgroups.

Growing number of studies describe gastrointestinal issues and early life antibiotic exposure in ADHD patients [16,38], elements that are associated with gut bacterial community. Therefore, we further analyzed the virulence factor and antibiotic resistance in ADHD patients' gut microbiota. The abundance of ARO ANT(9)-la correlated with the abundance of Prevotella_amnii, which was enriched in ADHD patients. Virulence factor and antibiotic resistance analyses could provide more integrated information for gut microbiota functions [39] although their significance validation was required in further studies.

Of note, the current study is the first to characterize the gut microbial community not only in ADHD patients but also in the I-ADHD and C-ADHD patient subgroups. We obtained a satisfactory classification with robust efficacy for distinguishing ADHD or C-ADHD patients from HCs. Here, we used species-based bacterial markers to calculate the AUC and achieved ideal values between ADHD vs. HC (0.84) and CADHD vs. HC (0.87). However, the AUC between I-ADHD and C-ADHD (0.76) indicated weak discriminatory validity. Therefore, these data further suggest that gut bacterial composition analysis in subgroup patients would obtain better differentiation. The similar bacterial patterns between I-ADHD patients and HCs may underline the smaller difference in ADHD vs. HCs than in C-ADHD vs. HCs. Together, the species used in the classification were potential microbiota markers for ADHD diagnosis, although further validation was required in larger cohorts.

The main strengths of the present study include metagenomic sequencing, subgroup analyses and larger sample size. Secondly, gut microbial virulence factor and antibiotic resistance were analyzed. Moreover, only medication-naïve patients with ADHD were recruited in the current study to exclude the effects of medication on gut microbiota. The same applies to psychiatric comorbidity that was also excluded. Given that the gut microbiome composition is highly correlated with diet pattern, a questionnaire including diet and defecation habits was collected from individual participants to assess dietary differences between groups. There are also some limitations in this study. First, our study could not obtain distinct gut profile 
information for HI-ADHD patients, due to the lack of enough HI-ADHD cases. Second, in line with a realworld scenario, the groups were not completely matched by sample size, sex, only child status, percentage of cesarean section and low birth weight. Although we found unmatched sex didn't alter the outcome that greater gut microbiota variation between C-ADHD and HC than between ADHD and HC. Third, we performed a cross-sectional study, and further longitudinal work should be conducted to further assess age- or medication-associated gut microbiota variation.

\section{Conclusions}

Our study characterized the distinct gut microbiota panel in ADHD and its subgroups of patients. We found more gut microbial alterations in C-ADHD patients than that in I-ADHD patients. We also identified several progressively enriched or decreased microbial taxa from $\mathrm{HC}$ to I-ADHD and C-ADHD, which is consistent with the clinical severity of ADHD. The predicted functional metabolic pathways according to these distinct bacterial taxa suggest compensatory host metabolism in ADHD patients. The current study provides new evidence to support that the microbiota-gut-brain axis is associated with ADHD. Further studies are required to evaluate these microbiota biomarkers in both ADHD and its phenotypically different clinical presentations.

\section{Methods}

\section{Subjects}

A total of 207 Chinese children and adolescents were recruited, including 98 ADHD patients $(Y=9.0$ years, $\mathrm{SD}=2.0)$ and $109 \mathrm{HCs}(\mathrm{Y}=8.9$ years, $\mathrm{SD}=1.8)$. All case and control samples were collected at $\mathrm{Xijing}$ Hospital, Shaanxi, China, between March 20, 2018, and February 27, 2020. ADHD patients were diagnosed and grouped using integrated visual and auditory continuous performance test (IVA-CPT) and structured diagnostic interview conducted according to the clinical Diagnostic and Statistical Manual of Mental Disorders, 4th edition (DSM-IV) by two experienced child psychiatrists. The numbers of the three different subgroups were 38 I-ADHD, 53 C-ADHD and 7 HI-ADHD, respectively. ADHD patients and HCs had to meet the following criteria: (1) age between 6 and 15 years old; (2) no use of any antibiotic treatment for at least three months before sample collection; and (3) no history of treatment with any medication for ADHD. The participants who had other psychiatric or neurological diseases were excluded. Children and adolescents whose IQs were below 70 according to the Wechsler Intelligence Scale for Children (WISC-V, http://wiscv.com/) were also excluded. In particular, in this study, a questionnaire including diet and defecation habits was collected from individual participants to assess dietary differences between the three groups. All of the participants provided written informed consent, and the study was approved by the ethics committee of Xijing Hospital, Fourth Military Medical University (ID: KY20182002-1). The present study was registered at ClinicalTrials.gov (ID: NCT03447223).

\section{DNA extraction and library construction}


Fresh feces (approximately $0.5 \mathrm{~g}$ ) were immediately moved into a sterilized collection tube by a sterilized wood stick from a clean toilet (MGI, China) by the parents of ADHD patients and HCs. The preservation method includes a reagent containing imidazolium-based ionic liquid [40]. After transport on dry ice, fecal samples were stored at $-80^{\circ} \mathrm{C}$ until DNA extraction. Total bacterial DNA from each fecal sample was extracted from $\sim 200 \mathrm{mg}$ of stool with the NucleoSpin ${ }^{\circledR}$ Soil kit (Macherey-Nagel, Germany) according the manufacturer's instructions. Qubit (Invitrogen, USA) and 1\% agarose gel electrophoresis were used to analyze the quality of DNA. The details of DNA library construction were as follows: $1 \mu \mathrm{g}$ genomic DNA was randomly fragmented by Covaris (Covaris, USA), and the fragmented DNA samples were tested by gel electrophotometry and then purified by a kit. The fragmented DNAs were combined with End Repair Mix, incubated at $20^{\circ} \mathrm{C}$ for $30 \mathrm{~min}$ and then purified by a kit. The repaired DNAs were combined with ATailing Mix and incubated at $37^{\circ} \mathrm{C}$ for $30 \mathrm{~min}$. Illumina adaptors were ligated to the Adenylated $3^{\prime} \mathrm{Ends}$ DNA, incubated at $16^{\circ} \mathrm{C}$ for $16 \mathrm{~h}$, and then purified with a kit. After selecting the correct insert size DNA fragments, several rounds of PCR amplification with PCR Primer Cocktail and PCR Master Mix were performed to enrich the adapter-ligated DNA fragments. The AxyPrePTM Mag PCR Clean-up kit (Axygen, USA) was used to purify DNA in all steps of DNA library construction. The final DNA libraries were assessed for the average insert size using an Agilent 2100 Bioanalyzer (Agilent Technologies, USA) and quantified by an ABI StepOnePlus Real-Time PCR system (Applied Biosystems, USA).

\section{Metagenomic sequencing}

Samples were sequenced on the Illumina HiSeq X Ten platform with an insert size of $300 \mathrm{bp}$ (paired end, 150 base pairs). Before further bioinformatic analysis, raw reads containing adaptor sequence, low quality (lower Q-score 20 rate more than $50 \%$ ) and ambiguous bases ( $\mathrm{N}$ base rate more than $5 \%$ ) were filtered out with SOAPnuke [41], and 1,486.5 Gb of high-quality PE reads for the 207 samples were acquired with an average of $7.18 \mathrm{~Gb}$ per sample (Table S12, Supporting Information). To remove human host DNA contamination, reads were aligned to the human genome reference (hg19) by SOAPaligner [42] (v2.22, parameters: -m 280 -x 420 -r 1 - I 32 -s 75 -c 0.9), and the mapping reads were discarded. The average rate of host contamination was $1.25 \pm 5.23 \%$.

\section{Metagenomic microbial community and functional profiling}

The profile of microbial composition for each sample was calculated using MetaPhIAn2 (v2.0) [43], which uses $\sim 1 \mathrm{M}$ unique clade-specific marker genes (including bacterial, archaeal, viral and eukaryotic) to estimate the relative abundance of bacterial taxa. The parameters of MetaPhIAn2 were set as '-nproc 10 -stat avg_g -ignore_viruses -ignore_eukaryotes -ignore_archaea'. Then, all sample profiles were merged using merge_metaphlan_tables.py.

Functional profiling for each sample was performed using HUMAnN2 [44] (v0.11.2). In brief, HUMAnN2 rapidly identified known microbial species in samples with MetaPhIAn2 and then constructed a customized pangenome database in which all genomes have been preconstructed and functionally annotated. Sample reads were mapped to this database with Bowtie2 [45], with the unmapped reads translated and mapped to a protein database (UniRef90) [46] with Diamond [47]. Finally, all mapping 
reads were used to estimate gene family abundance and then annotated to metabolic enzymes to reconstruct and quantify metabolic pathways (MetaCyc) [48]. HUMAnN2 was run by the default parameters. All sample profiles were merged and renormalized using humann2_join_tables and humann2_renorm_table, respectively.

Virulence factor proteins and AROs were analyzed according to methods previously reported [39]. In brief, protein reference databases were downloaded from the Virulence Factors Database (VFDB, v201806) [49] and Comprehensive Antibiotic Resistance Database (CARD, v2.0.1) [50], respectively. Then, high quality sequencing reads were aligned to protein reference databases using DIAMOND [47] and the parameters were set as '-threads 10 -max-target-seqs 1 -outfmt 6'. If the paired-end reads aligned to the same protein and the identity was more than $70 \%$, E value lower than $1 \mathrm{e}-05$, they were considered to be vailed alignment. Finally, quantified as counts per million (CMP) was used to calculate the proteins abundances: the raw valid counts (number of valid alignments) divided by the library sizes (total high quality sequencing reads of each sample) and multiplied by one million.

Taxa, gene families, pathways, virulence factor proteins and AROs which presented in less than $10 \%$ samples were discarded in further analysis.

\section{Statistical analysis}

Alpha diversity of samples was estimated by Shannon diversity at the gene family level. Beta diversity between samples was estimated by Bray-Curtis distance at the gene family level via the 'vegdist' function in the vegan R package. PERMANOVA based on the Bray-Curtis distance matrix was performed via the 'adonis' function from the R package vegan, and the permuted $p$ value was obtained by 9,999 permutations. Supervised analysis with spare PLS-DA was performed using the mixOmics package in R. Differential relative abundances of taxa and pathways were detected by the Wilcoxon rank-sum test and LEfSe [51], and the significance levels were $p$ value $<0.05$ and LDA $>2$. The correlations between the relative abundance of species and metabolic pathways or host characteristics were calculated by Spearman's rank correlation coefficient and visualized by heatmap in R using the ComplexHeatmap package. Because of the high correlation between species and metabolic pathways, a method reported previously [52] was used to determine the significance $p$ value $\left(0.05 /(25 \times 399)=5.01 \times 10^{-6}\right)$. Virulence factor proteins and AROs were considered significant different between ADHD patients and HCs if $p$ value of Fisher's exact test and Wilcoxon rank sum test both less than 0.05 . Odds ratio (OR) and $95 \%$ were calculated by logistic regression in R and adjusted age, sex and BMI. Biomarkers used to discriminate ADHD patients and HCs were identified based on a random forest model (randomForest 4.6-14 package) using the relative abundance profile at the species level [53], and the result was assessed by receiver operating characteristic (ROC) curves using the pROC package in R. The AUC is a convenient tool for comparing and validating classifiers, and values of 0.90-1.00 are excellent, $0.80-0.89$ are good, $0.70-$ 0.79 are fair, and $<0.70$ are poor [54].

\section{Abbreviations}


ADHD:Attention-deficit/hyperactivity disorder; HCs:healthy controls; BMI:body mass index; IQ:intelligence quotient; DSM:Diagnostic and Statistical Manual of Mental Disorders; PERMANOVA:permutational multivariate analysis of variance; ARO:antibiotic resistance ontology; RF:Random forest; VFDB:Virulence Factors Database; CARD:Comprehensive Antibiotic Resistance Database; CMP:counts per million

\section{Declarations}

\section{Availability of data and materials}

The metagenomic shotgun sequencing data for all samples have been deposited in the CNGB Nucleotide Sequence Archive (CNSA) under accession code CNP0000729. Other data that support the findings of this study are available within the paper and its Supplementary Information files or from the corresponding author upon reasonable request.

\section{Ethics approval and consent to participate}

This study was approved by the ethics committee of Xijing Hospital, Fourth Military Medical University (ID: KY20182002-1). All participants were informed about the purpose of this study and provided written informed consent.

\section{Consent for publication}

Not applicable.

\section{Competing interests}

Luis Augusto Rohde has received grant or research support from, served as a consultant to, and served on the speakers' bureau of Medice, Novartis/Sandoz and Shire/Takeda in the last three years. The ADHD and Juvenile Bipolar Disorder Outpatient Programs chaired by Dr Rohde have received unrestricted educational and research support from the following pharmaceutical companies in the last three years: Novartis/Sandoz and Shire/Takeda. Dr Rohde has received authorship royalties from Oxford Press and ArtMed and travel grants from Shire to take part in the 2018 APA annual meeting and from Novartis to take part of the 2017 AACAP annual meeting.

\section{Funding}

This work was supported by the National Natural Science Foundation of China (Grant No. 81671184), the Natural Science Fund for Distinguished Young Scholars of Shaanxi Province (2019JC-24), the 
Fundamental Research Funds for the Central Universities of China (xtr022019005), and the Clinical Research Award of the First Affiliated Hospital of Xi'an Jiaotong University (XJTU1AF-CRF-2018-003).

\section{Authors' contributions}

Y.L., H.T.S., Y.F.H., and A.Q.Y. contributed equally to this study. L.Z.X., Y.L., X.S., and X.F.X. conceived, designed, and supervised the study. H.T.S., P.W., M.T., C.W., F.F.J., A.Q.Y., and X.S. recruited the subjects and collected the fecal samples. Y.F.H., X.F.X., J.Y.Z., and S.L.S. participated in the data interpretation and statistical analysis. Y.L. and Y.F.H. drafted the manuscript. L.Z.X., L.A.R., J.H., D.D.Q., and L.X.Z. checked and revised the manuscript. All authors read and approved the final manuscript.

\section{Acknowledgments}

We thank Qian Kou, Jiayan Wang, Xiaolan Wang and Jing Xi from the Department of Pediatrics, Xijing Hospital, the Fourth Military Medical University for their support in data collection.

\section{References}

1. Posner J, Polanczyk GV, Sonuga-Barke E. Attention-deficit hyperactivity disorder. Lancet. 2020;395:450-62.

2. Polanczyk G, de Lima MS, Horta BL, Biederman J, Rohde LA. The worldwide prevalence of ADHD: a systematic review and metaregression analysis. Am J Psychiatry. 2007;164:942-8.

3. Willcutt EG, Nigg JT, Pennington BF, Solanto MV, Rohde LA, Tannock R, Loo SK, Carlson CL, McBurnett K, Lahey BB. Validity of DSM-IV attention deficit/hyperactivity disorder symptom dimensions and subtypes. J Abnorm Psychol. 2012;121:991-1010.

4. Dalsgaard S, Ostergaard SD, Leckman JF, Mortensen PB, Pedersen MG. Mortality in children, adolescents, and adults with attention deficit hyperactivity disorder: a nationwide cohort study. Lancet. 2015;385:2190-6.

5. Demontis D, Walters RK, Martin J, Mattheisen M, Als TD, Agerbo E, Baldursson G, Belliveau R, BybjergGrauholm J, Baekvad-Hansen $\mathrm{M}$, et al. Discovery of the first genome-wide significant risk loci for attention deficit/hyperactivity disorder. Nat Genet. 2019;51:63-75.

6. Cenit MC, Nuevo IC, Codoner-Franch P, Dinan TG, Sanz Y. Gut microbiota and attention deficit hyperactivity disorder: new perspectives for a challenging condition. Eur Child Adolesc Psychiatry. 2017;26:1081-92.

7. Lai WT, Deng WF, Xu SX, Zhao J, Xu D, Liu YH, Guo YY, Wang MB, He FS, Ye SW, et al: Shotgun metagenomics reveals both taxonomic and tryptophan pathway differences of gut microbiota in major depressive disorder patients. Psychol Med 2019:1-12. 
8. Hu S, Li A, Huang T, Lai J, Li J, Sublette ME, Lu H, Lu Q, Du Y, Hu Z, et al. Gut Microbiota Changes in Patients with Bipolar Depression. Adv Sci (Weinh). 2019;6:1900752.

9. Zheng P, Zeng B, Liu M, Chen J, Pan J, Han Y, Liu Y, Cheng K, Zhou C, Wang H, et al. The gut microbiome from patients with schizophrenia modulates the glutamate-glutamine-GABA cycle and schizophrenia-relevant behaviors in mice. Sci Adv. 2019;5:eaau8317.

10. Sharon G, Cruz NJ, Kang DW, Gandal MJ, Wang B, Kim YM, Zink EM, Casey CP, Taylor BC, Lane CJ, et al. Human Gut Microbiota from Autism Spectrum Disorder Promote Behavioral Symptoms in Mice. Cell. 2019;177:1600-18 e1617.

11. Tengeler AC, Dam SA, Wiesmann M, Naaijen J, van Bodegom M, Belzer C, Dederen PJ, Verweij V, Franke B, Kozicz T, et al. Gut microbiota from persons with attention-deficit/hyperactivity disorder affects the brain in mice. Microbiome. 2020;8:44.

12. Vuong HE, Yano JM, Fung TC, Hsiao EY. The Microbiome and Host Behavior. Annu Rev Neurosci. 2017;40:21-49.

13. Cryan JF, O'Riordan KJ, Cowan CSM, Sandhu KV, Bastiaanssen TFS, Boehme M, Codagnone MG, Cussotto S, Fulling C, Golubeva AV, et al. The Microbiota-Gut-Brain Axis. Physiol Rev. 2019;99:18772013.

14. Curran EA, Khashan AS, Dalman C, Kenny LC, Cryan JF, Dinan TG, Kearney PM. Obstetric mode of delivery and attention-deficit/hyperactivity disorder: a sibling-matched study. Int J Epidemiol. 2016;45:532-42.

15. Adesman A, Soled D, Rosen L. Formula Feeding as a Risk Factor for Attention-Deficit/Hyperactivity Disorder: Is Bisphenol A Exposure a Smoking Gun? J Dev Behav Pediatr. 2017;38:545-51.

16. Slykerman RF, Coomarasamy C, Wickens K, Thompson JMD, Stanley TV, Barthow C, Kang J, Crane J, Mitchell EA. Exposure to antibiotics in the first 24 months of life and neurocognitive outcomes at 11 years of age. Psychopharmacology. 2019;236:1573-82.

17. Pelsser LM, Frankena K, Toorman J, Rodrigues Pereira R. Diet and ADHD, Reviewing the Evidence: $A$ Systematic Review of Meta-Analyses of Double-Blind Placebo-Controlled Trials Evaluating the Efficacy of Diet Interventions on the Behavior of Children with ADHD. PLoS One. 2017;12:e0169277.

18. Aarts E, Ederveen THA, Naaijen J, Zwiers MP, Boekhorst J, Timmerman HM, Smeekens SP, Netea MG, Buitelaar JK, Franke B, et al. Gut microbiome in ADHD and its relation to neural reward anticipation. PLoS One. 2017;12:e0183509.

19. Jiang HY, Zhou YY, Zhou GL, Li YC, Yuan J, Li XH, Ruan B. Gut microbiota profiles in treatment-naive children with attention deficit hyperactivity disorder. Behav Brain Res. 2018;347:408-13.

20. Prehn-Kristensen A, Zimmermann A, Tittmann L, Lieb W, Schreiber S, Baving L, Fischer A. Reduced microbiome alpha diversity in young patients with ADHD. PLoS One. 2018;13:e0200728.

21. Wang LJ, Yang CY, Chou WJ, Lee MJ, Chou MC, Kuo HC, Yeh YM, Lee SY, Huang LH, Li SC. Gut microbiota and dietary patterns in children with attention-deficit/hyperactivity disorder. Eur Child Adolesc Psychiatry. 2020;29:287-97. 
22. Szopinska-Tokov J, Dam S, Naaijen J, Konstanti P, Rommelse N, Belzer C, Buitelaar J, Franke B, Aarts E, Arias Vasquez A: Investigating the Gut Microbiota Composition of Individuals with Attention-
Deficit/Hyperactivity Disorder and Association with Symptoms. Microorganisms 2020, 8.

23. Wan L, Ge WR, Zhang S, Sun YL, Wang B, Yang G. Case-Control Study of the Effects of Gut Microbiota Composition on Neurotransmitter Metabolic Pathways in Children With Attention Deficit Hyperactivity Disorder. Front Neurosci. 2020;14:127.

24. Rakoff-Nahoum S, Foster KR, Comstock LE. The evolution of cooperation within the gut microbiota. Nature. 2016;533:255-9.

25. Tillisch K, Mayer EA, Gupta A, Gill Z, Brazeilles R, Le Neve B, van Hylckama Vlieg JET, Guyonnet D, Derrien M, Labus JS. Brain Structure and Response to Emotional Stimuli as Related to Gut Microbial Profiles in Healthy Women. Psychosom Med. 2017;79:905-13.

26. Daubner SC, Le T, Wang S. Tyrosine hydroxylase and regulation of dopamine synthesis. Arch Biochem Biophys. 2011;508:1-12.

27. van der Kooij MA, Glennon JC. Animal models concerning the role of dopamine in attention-deficit hyperactivity disorder. Neurosci Biobehav Rev. 2007;31:597-618.

28. Swanson JM, Kinsbourne M, Nigg J, Lanphear B, Stefanatos GA, Volkow N, Taylor E, Casey BJ, Castellanos FX, Wadhwa PD. Etiologic subtypes of attention-deficit/hyperactivity disorder: brain imaging, molecular genetic and environmental factors and the dopamine hypothesis. Neuropsychol Rev. 2007;17:39-59.

29. Park S, Cho SC, Kim JW, Shin MS, Yoo HJ, Oh SM, Han DH, Cheong JH, Kim BN. Differential perinatal risk factors in children with attention-deficit/hyperactivity disorder by subtype. Psychiatry Res. 2014;219:609-16.

30. Magistretti PJ, Allaman I. A cellular perspective on brain energy metabolism and functional imaging. Neuron. 2015;86:883-901.

31. Vital M, Howe A, Bergeron N, Krauss RM, Jansson JK, Tiedje JM. Metagenomic Insights into the Degradation of Resistant Starch by Human Gut Microbiota. Appl Environ Microbio/ 2018, 84.

32. Ostojic SM. Inadequate Production of H2 by Gut Microbiota and Parkinson Disease. Trends Endocrinol Metab. 2018;29:286-8.

33. Petit E, LaTouf WG, Coppi MV, Warnick TA, Currie D, Romashko I, Deshpande S, Haas K, AlveloMaurosa JG, Wardman C, et al. Involvement of a bacterial microcompartment in the metabolism of fucose and rhamnose by Clostridium phytofermentans. PLoS One. 2013;8:e54337.

34. Schretter CE, Vielmetter J, Bartos I, Marka Z, Marka S, Argade S, Mazmanian SK. A gut microbial factor modulates locomotor behaviour in Drosophila. Nature. 2018;563:402-6.

35. dos Santos FS, da Silva LA, Pochapski JA, Raczenski A, da Silva WC, Grassiolli S, Malfatti CR. Effects of l-arginine and creatine administration on spatial memory in rats subjected to a chronic variable stress model. Pharm Biol. 2014;52:1033-8.

36. Zheng P, Yu B, He J, Yu J, Mao X, Luo Y, Luo J, Huang Z, Tian G, Zeng Q, et al. Arginine metabolism and its protective effects on intestinal health and functions in weaned piglets under oxidative stress 
induced by diquat. Br J Nutr. 2017;117:1495-502.

37. Landaas ET, Aarsland TI, Ulvik A, Halmoy A, Ueland PM, Haavik J. Vitamin levels in adults with ADHD. BJPsych Open. 2016;2:377-84.

38. Ming X, Chen N, Ray C, Brewer G, Kornitzer J, Steer RA. A Gut Feeling: A Hypothesis of the Role of the Microbiome in Attention-Deficit/Hyperactivity Disorders. Child Neurol Open. 2018;5:2329048 × 18786799.

39. Vich Vila A, Imhann F, Collij V, Jankipersadsing SA, Gurry T, Mujagic Z, Kurilshikov A, Bonder MJ, Jiang $\mathrm{X}$, Tigchelaar EF, et al: Gut microbiota composition and functional changes in inflammatory bowel disease and irritable bowel syndrome. Sci Transl Med 2018, 10.

40. Hao L, Xia Z, Yang H, Wang J, Han M. Ionic liquid-based reagents improve the stability of midterm fecal sample storage. J Microbiol Methods. 2017;139:68-73.

41. Chen Y, Chen Y, Shi C, Huang Z, Zhang Y, Li S, Li Y, Ye J, Yu C, Li Z, et al. SOAPnuke: a MapReduce acceleration-supported software for integrated quality control and preprocessing of high-throughput sequencing data. Gigascience. 2018;7:1-6.

42. Li R, Li Y, Kristiansen K, Wang J. SOAP: short oligonucleotide alignment program. Bioinformatics. 2008;24:713-4.

43. Truong DT, Franzosa EA, Tickle TL, Scholz M, Weingart G, Pasolli E, Tett A, Huttenhower C, Segata N. MetaPhIAn2 for enhanced metagenomic taxonomic profiling. Nat Methods. 2015;12:902-3.

44. Franzosa EA, Mclver LJ, Rahnavard G, Thompson LR, Schirmer M, Weingart G, Lipson KS, Knight R, Caporaso JG, Segata N, Huttenhower C. Species-level functional profiling of metagenomes and metatranscriptomes. Nat Methods. 2018;15:962-8.

45. Langmead B, Salzberg SL. Fast gapped-read alignment with Bowtie 2. Nat Methods. 2012;9:357-9.

46. Suzek BE, Wang Y, Huang H, McGarvey PB, Wu CH, UniProt C. UniRef clusters: a comprehensive and scalable alternative for improving sequence similarity searches. Bioinformatics. 2015;31:926-32.

47. Buchfink B, Xie C, Huson DH. Fast and sensitive protein alignment using DIAMOND. Nat Methods. 2015;12:59-60.

48. Caspi R, Billington R, Ferrer L, Foerster H, Fulcher CA, Keseler IM, Kothari A, Krummenacker M, Latendresse M, Mueller LA, et al. The MetaCyc database of metabolic pathways and enzymes and the BioCyc collection of pathway/genome databases. Nucleic Acids Res. 2016;44:D471-80.

49. Chen L, Zheng D, Liu B, Yang J, Jin Q. VFDB 2016: hierarchical and refined dataset for big data analysis-10 years on. Nucleic Acids Res. 2016;44:D694-7.

50. McArthur AG, Waglechner N, Nizam F, Yan A, Azad MA, Baylay AJ, Bhullar K, Canova MJ, De Pascale G, Ejim L, et al. The comprehensive antibiotic resistance database. Antimicrob Agents Chemother. 2013;57:3348-57.

51. Segata N, Izard J, Waldron L, Gevers D, Miropolsky L, Garrett WS, Huttenhower C. Metagenomic biomarker discovery and explanation. Genome Biol. 2011;12:R60. 
52. Li J, Ji L. Adjusting multiple testing in multilocus analyses using the eigenvalues of a correlation matrix. Heredity. 2005;95:221-7.

53. Feng Q, Liang S, Jia H, Stadlmayr A, Tang L, Lan Z, Zhang D, Xia H, Xu X, Jie Z, et al. Gut microbiome development along the colorectal adenoma-carcinoma sequence. Nat Commun. 2015;6:6528.

54. Cicchetti DV. The precision of reliability and validity estimates re-visited: distinguishing between clinical and statistical significance of sample size requirements. J Clin Exp Neuropsychol. 2001;23:695-700.

Figures

a

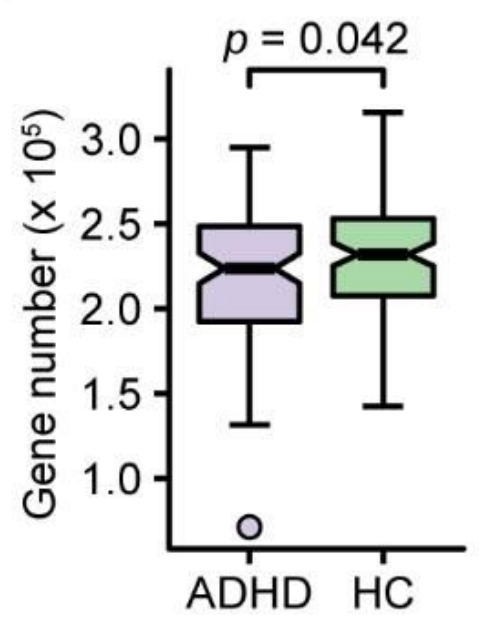

b
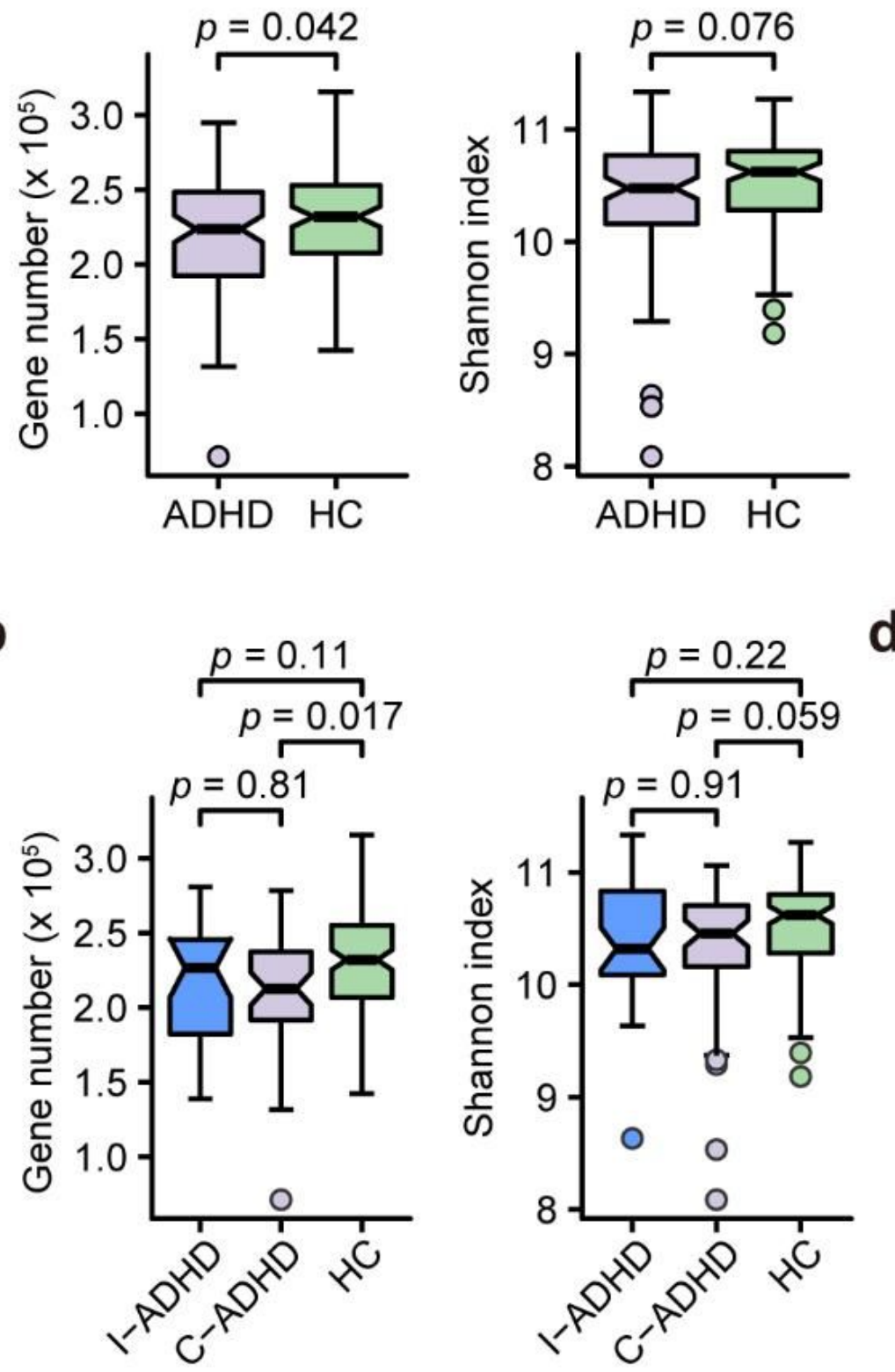

C
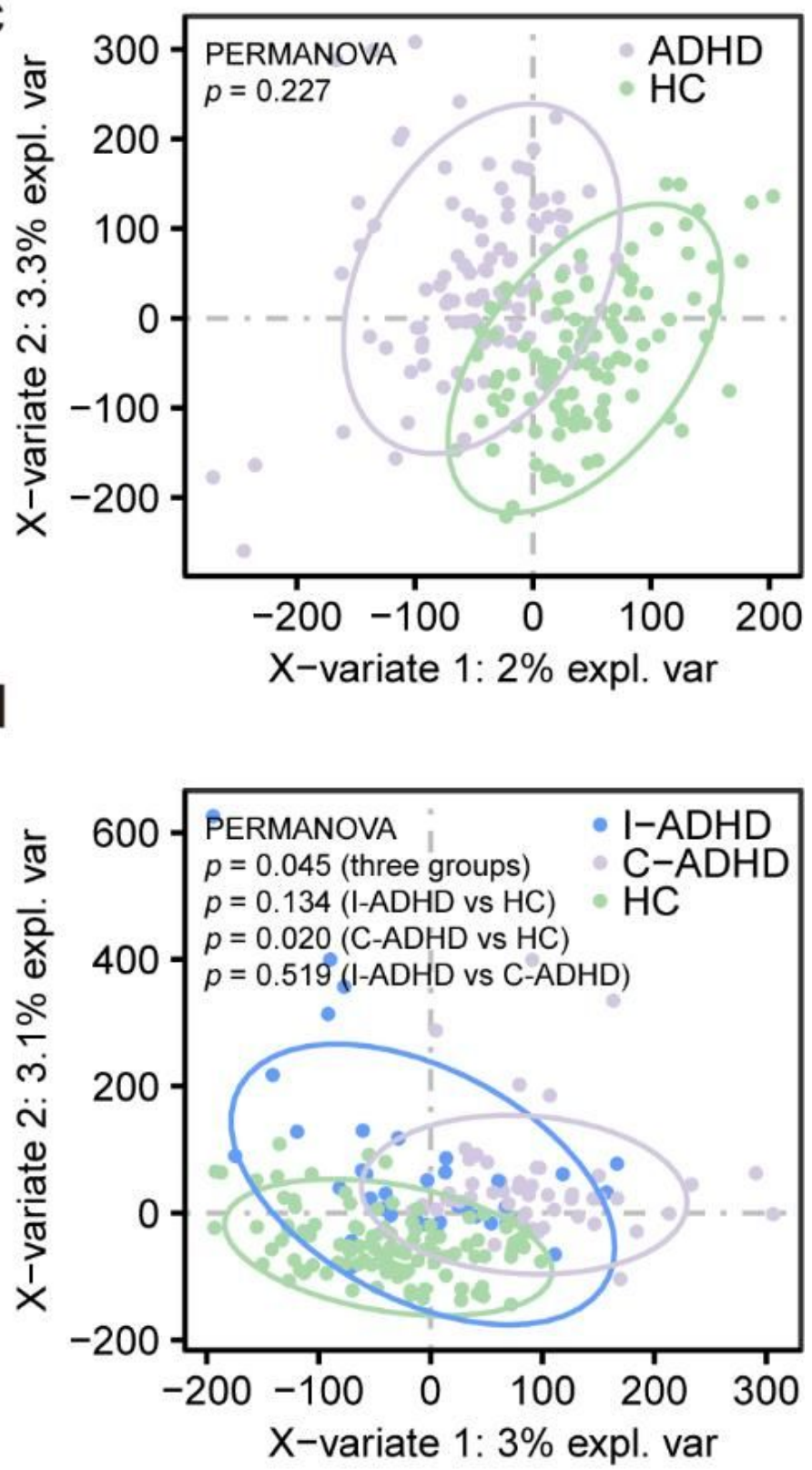

Figure 1 
Alpha and beta diversity analyses in ADHD patients and HCs. (a, b) Gene count and alpha diversity (Shannon index) in ADHD (a) or subgroup patients (b) and HCs. The Wilcoxon rank-sum test was used to determine significance. (c, d) Supervised analysis with spare PLS-DA in ADHD (c) or subgroup (d) patients at the gene level. PERMANOVA calculation based on the Bray Curtis distance at the gene level.

a

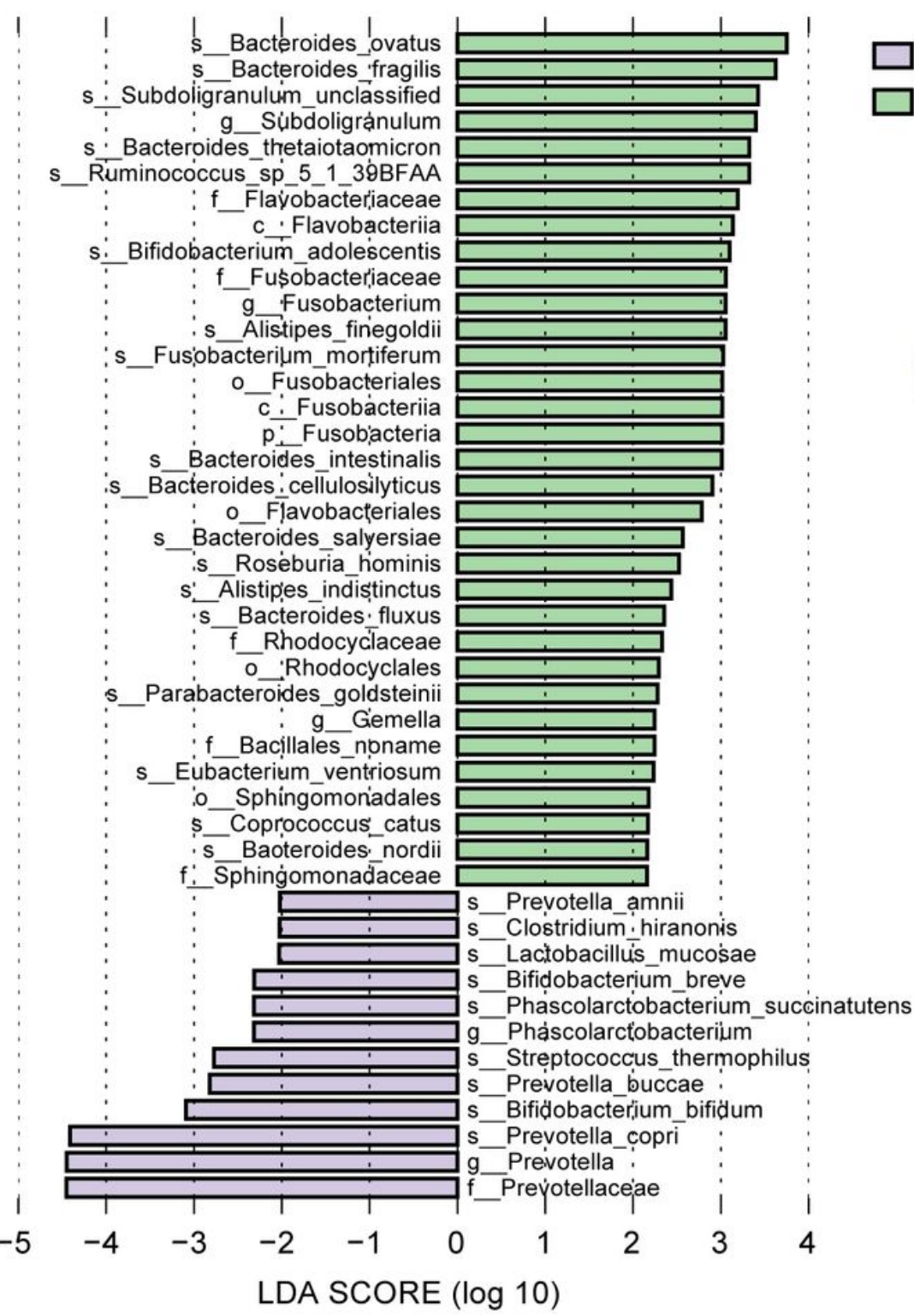

b

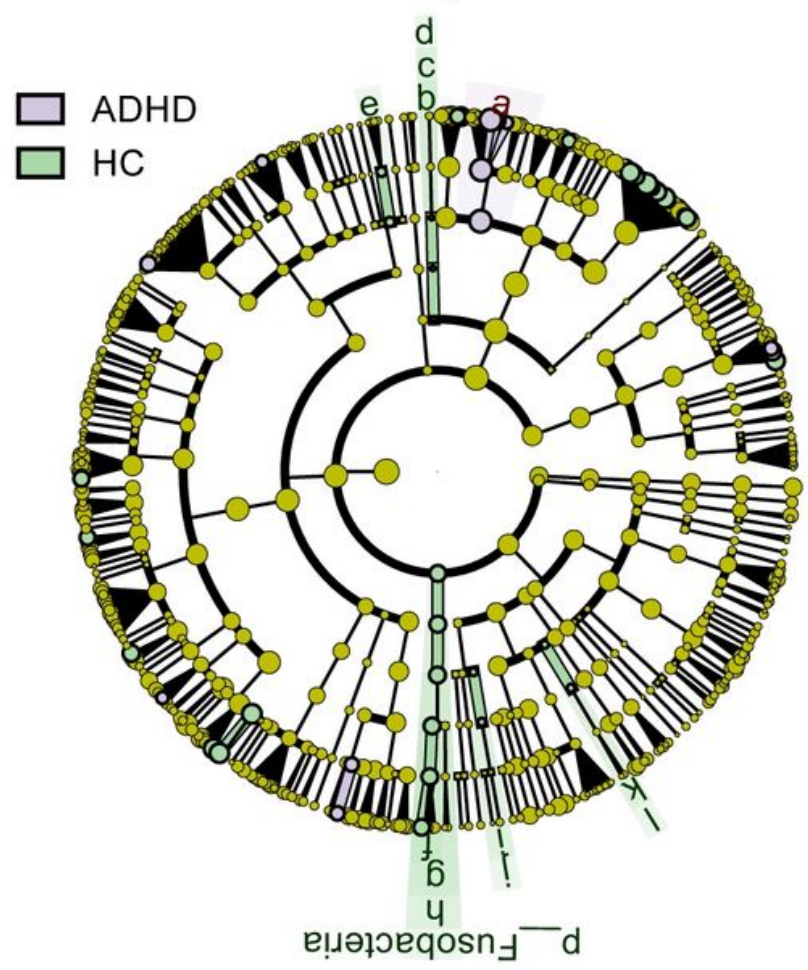

Figure 2

Significantly different taxa between ADHD patients and HCs. (a) LDA effect size analysis identified significantly different taxa between ADHD patients and HCs. The LDA scores $(\log 10)>2$ and $p<0.05$ are shown. A negative LDA score indicated enrichment in ADHD patients (purple), while a positive LDA score indicated enrichment in HCs (green). Bar length indicates the effect size of each taxon. (b) Taxonomic cladogram obtained from LEfSe analysis. The circles from inside to outside represent different classification levels, and the size of each dot is proportional to its relative abundance. The colored taxa 
represent significantly different taxa between ADHD patients and HCs. Purple, ADHD-enriched; Green, HCenriched.

a

C-ADHD vs HC

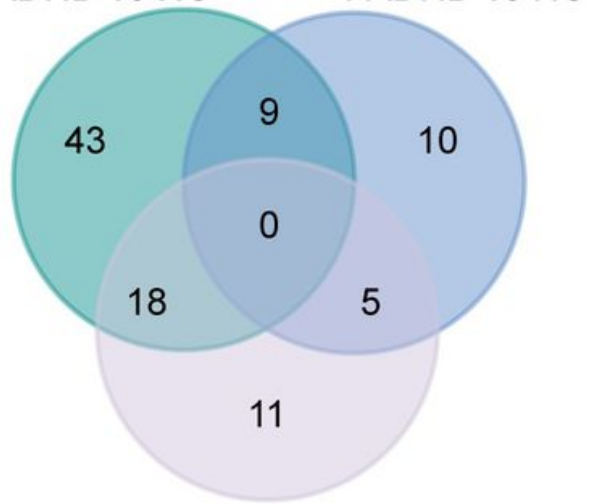

I-ADHD vs C-ADHD

C

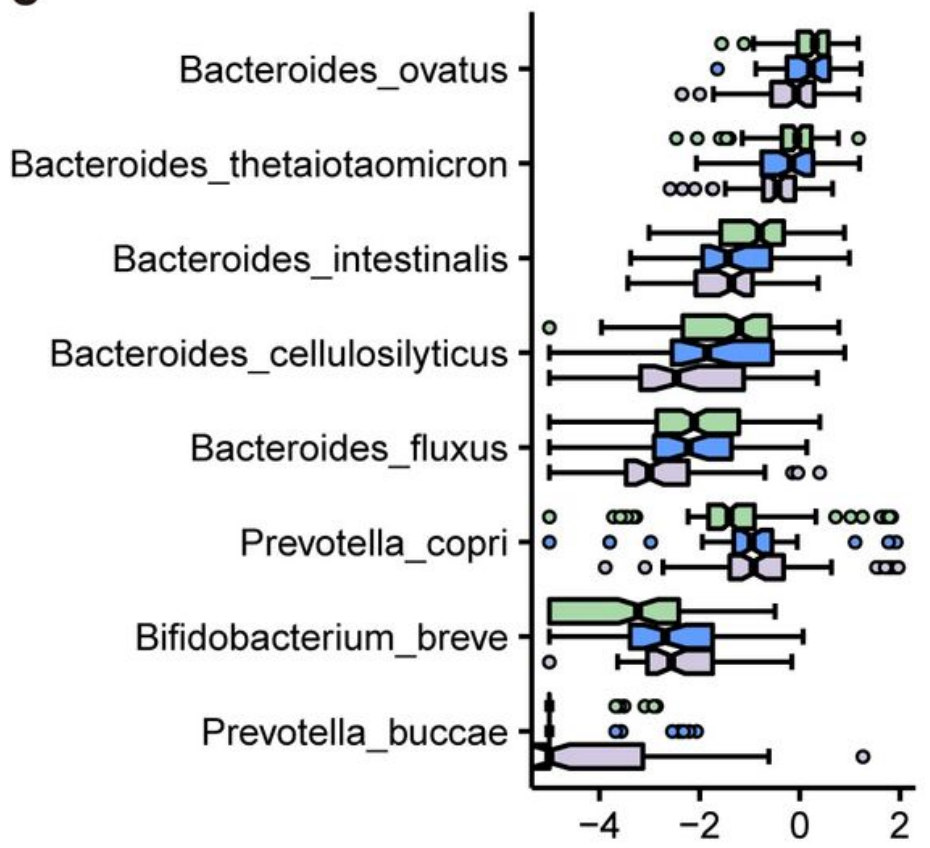

Relative abundance $\left(\log _{10}\right)$

追 $\mathrm{HC}$ 追 I-ADHD 追 C-ADHD b

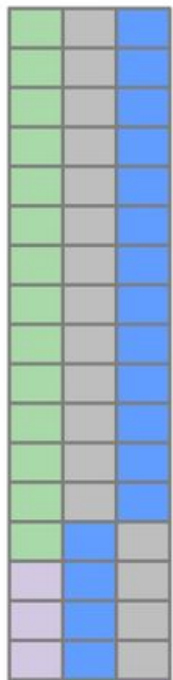

s_Bacteroides_cellulosilyticus

s_Bacteroides_fluxus

s_Bacteroides_nordii

s_Bacteroides_ovatus

s_Lachnospiraceae_bacterium_7_1_58FAA

o_ Rhizobiales

g_Bilophila

s_Bilophila_wadsworthia

$f$-Oscillospiraceae

g_Oscillibacter

s Oscillibacter unclassified

g__ubdoligranulum

s_Subdoligranulum_unclassified

$s$ - Bacteroides intestinalis

s - Bifidobacterium bifidum

s_Bifidobacterium breve

s_Prevotella_copri

s_Roseburia_intestinalis

$f$ Fusobacteriaceae

g- Fusobacterium

$\mathrm{s}$ - Fusobacterium mortiferum

s Ruminococcus sp 5 1 39BFAA

$\mathrm{S}$ _Parabacteroides_goldsteinii

g- Acidaminococcus

s_Acidaminococcus_intestini

s - Bifidobacterium catenulatum

s B Bifidobacterium_pseudocatenulatum

$f$ Listeriaceae

g_Listeria

$\mathrm{s}$ Listeria marthii

$f$ Prevotellaceae

f_Veillonellaceae

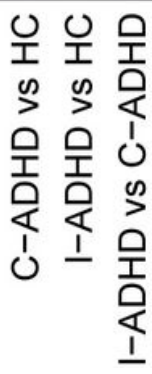

Enrichment

I-ADHD

C-ADHD

$\mathrm{HC}$

NS

Figure 3

The gut microbial composition of C-ADHD is different from that of I-ADHD and HC. (a) Venn diagram of significantly different taxa among different comparisons. (b) Heat map of sharing significantly different taxa among different comparisons. LEfSe analysis was used to detect significantly different taxa in (a) and (b). (c) Boxplot of significantly different species among HC, I-ADHD, and C-ADHD. Significant differences among groups were evaluated using the Jonckheere-Terpstra test and adjusted by the Benjamin-Hochberg $(\mathrm{BH})$ method $(\mathrm{p}<0.05)$. 


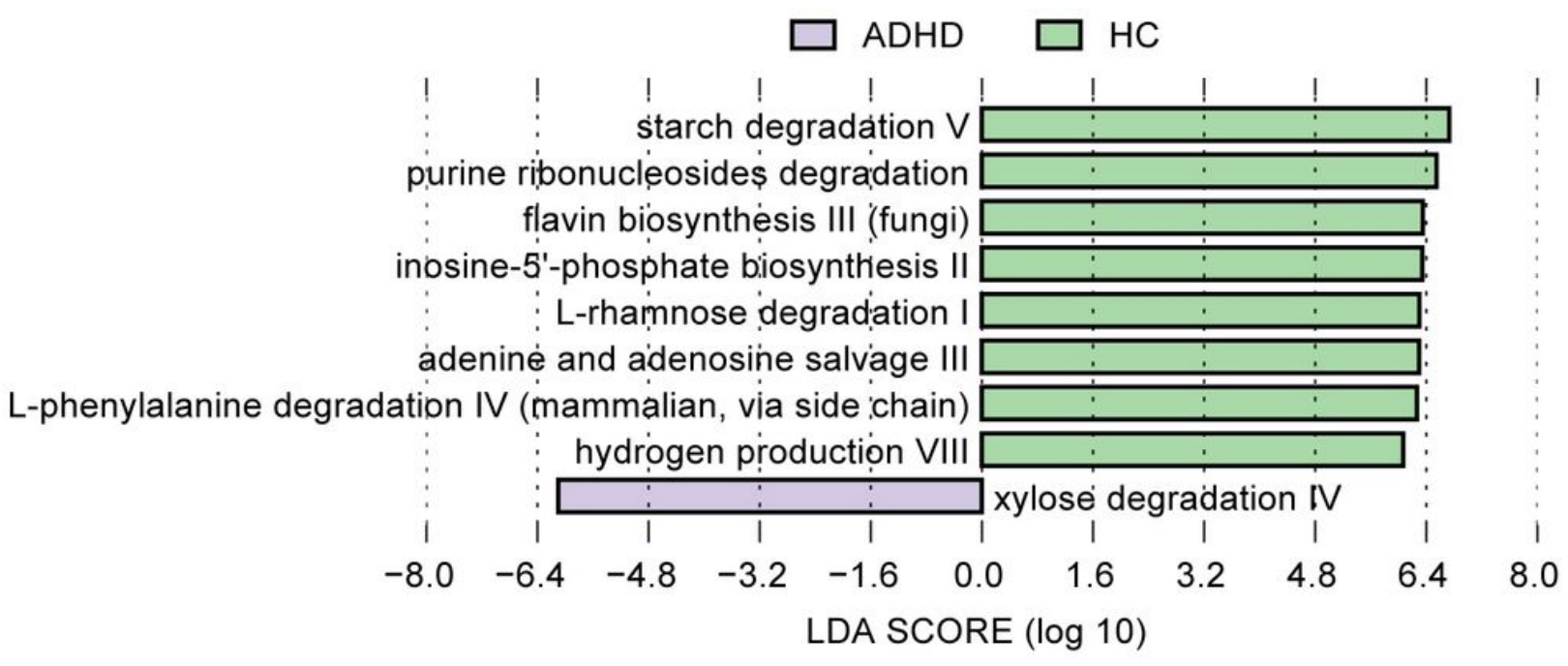

b L-phenylalanine degradation IV (mammálian, via side chain)

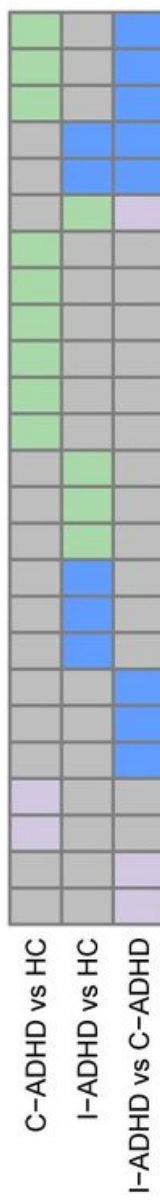

superpathway of GDP-mannose-derived O-antigen building blocks biosynthesis pantothenate and coenzyme A biosynthesis III L-histidine biosynthesis superpathway of $\mathrm{L}$-lysine, $\mathrm{L}$-threonine and $\mathrm{L}$-methionine biosynthesis I anhydromuropeptides recycling superpathway of pyrimidine ribonucleotides de novo biosynthesis $\mathrm{L}$-arginine biosynthesis III (via N-acetyl-L-citrulline) L-rhamnose degradation I flavin biosynthesis III (fungi) inosine-5-phosphate biosynthesis I superpathway of pyridoxal 5-phosphate biosynthesis and salvage NAD salvage pathway I purine ribonucleosides degradation GDP-mannose biosynthesis stachyose degradation superpathway of tetrahydrofolate biosynthesis and salvage superpathway of tetrahydrofolate biosynthesis superpathway of heme biosynthesis from glycine L-citrulline biosynthesis heme biosynthesis I (aerobic) superpathway of pyrimidine ribonucleosides degradation $\mathrm{S}$-adenosyl- $\mathrm{L}$-methionine cycle I xylose degradation IV putrescine biosynthesis IV adenosylcobalamin salvage from cobinamide I

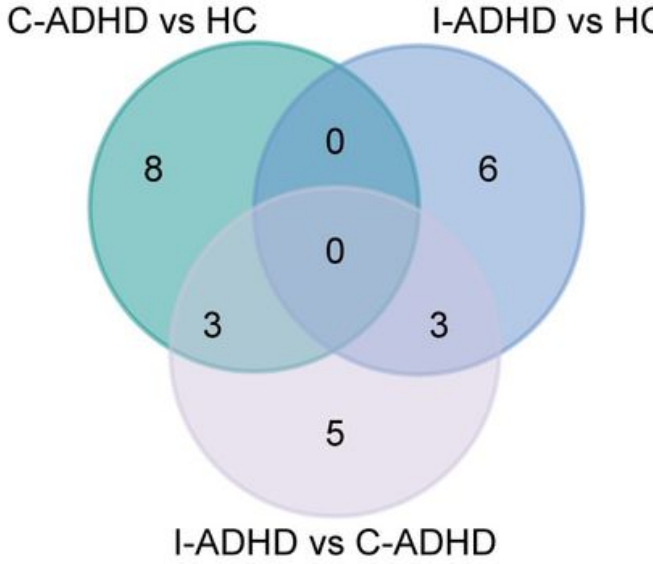

Figure 4

Gut microbial functions of C-ADHD are different from those of I-ADHD and HC. (a) Significantly different pathways between ADHD subgroup patients and HCs. The LDA scores (log 10) $>2$ and $p<0.05$ are shown. Bar length indicates the effect size of each species. (b) Heat map of sharing significantly different pathways among different comparisons by LEfSe analysis. (c) Venn diagram of significantly different pathways among different comparisons. 


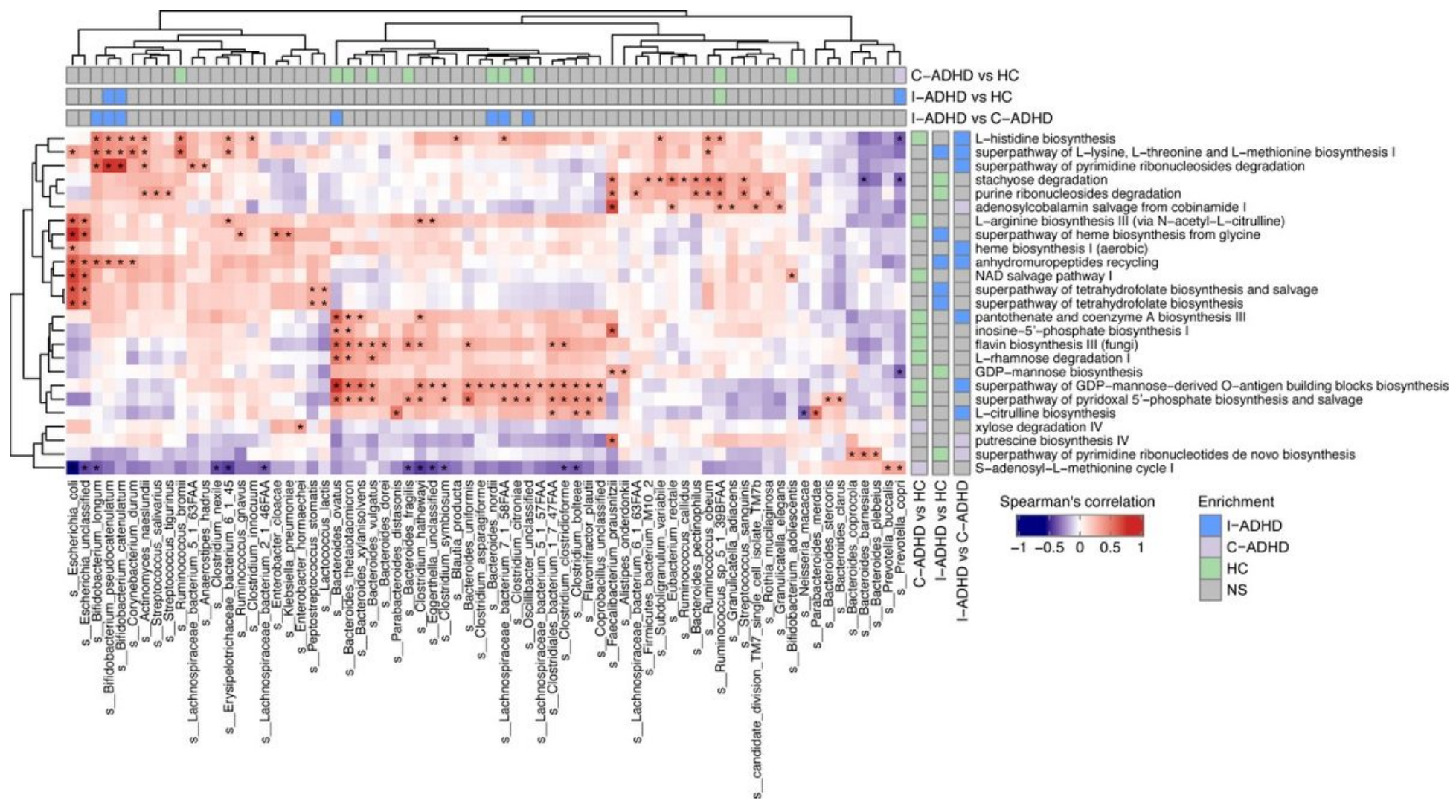

Figure 5

Correlation between gut microbial composition and function. Heat map of Spearman's correlation coefficient between the relative abundance of species and pathways (red and purple for positive and negative correlation, respectively). Species and pathway enrichment direction in different comparisons are shown on the top and left, respectively. Blue, I-ADHD-enriched; purple, C-ADHD-enriched; green, HCenriched; gray, no significant difference. ' ${ }^{\prime}$ ' denotes $p<0.001$. 


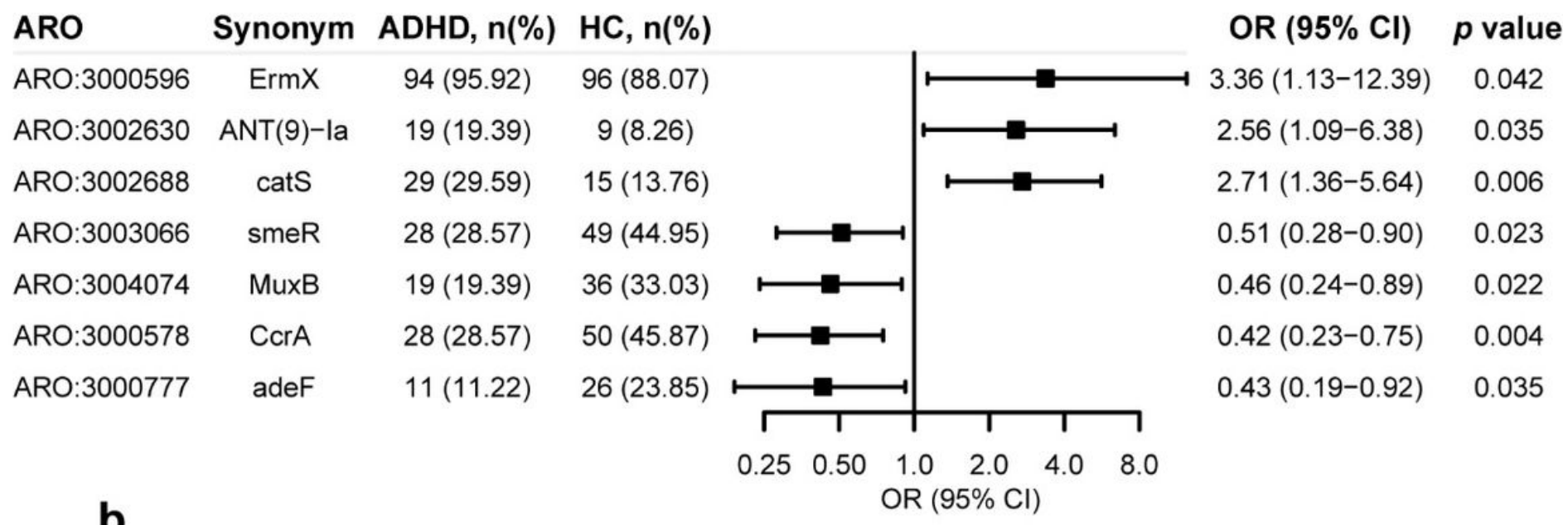

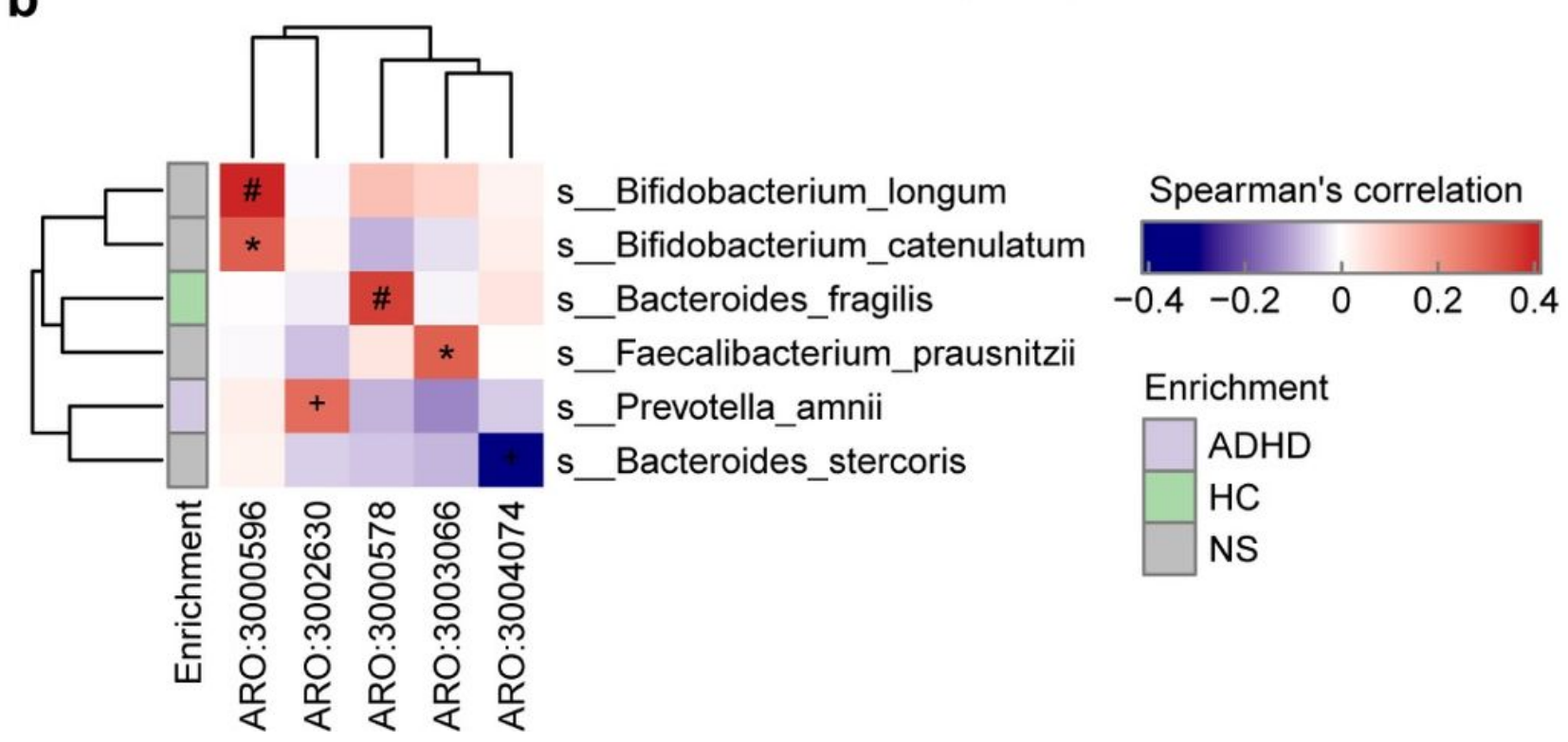

Figure 6

Antibiotic resistance ontology (ARO) between ADHD and HC. (a) Odd ratios of ARO in ADHD patients and $\mathrm{HCs}$. Black squares and bars represent odds ratios and $95 \% \mathrm{Cl}$, respectively, and were adjusted age, sex and $\mathrm{BMI}$ by logistic regression. (b) Correlation between gut microbial composition and ARO. Heat map of spearman's correlation coefficient between relative abundance of species and ARO (red and purple for positive and negative correlation, respectively). Species enrichment direction was shown on left. Purple, ADHD-enriched; green, HC-enriched; gray, no significant difference. ' + ' denotes $\mathrm{P}<0.05$; ' ${ }^{\prime}$ ' denotes $\mathrm{P}<$ 0.01 , '\#' denotes $\mathrm{P}<0.001$. 


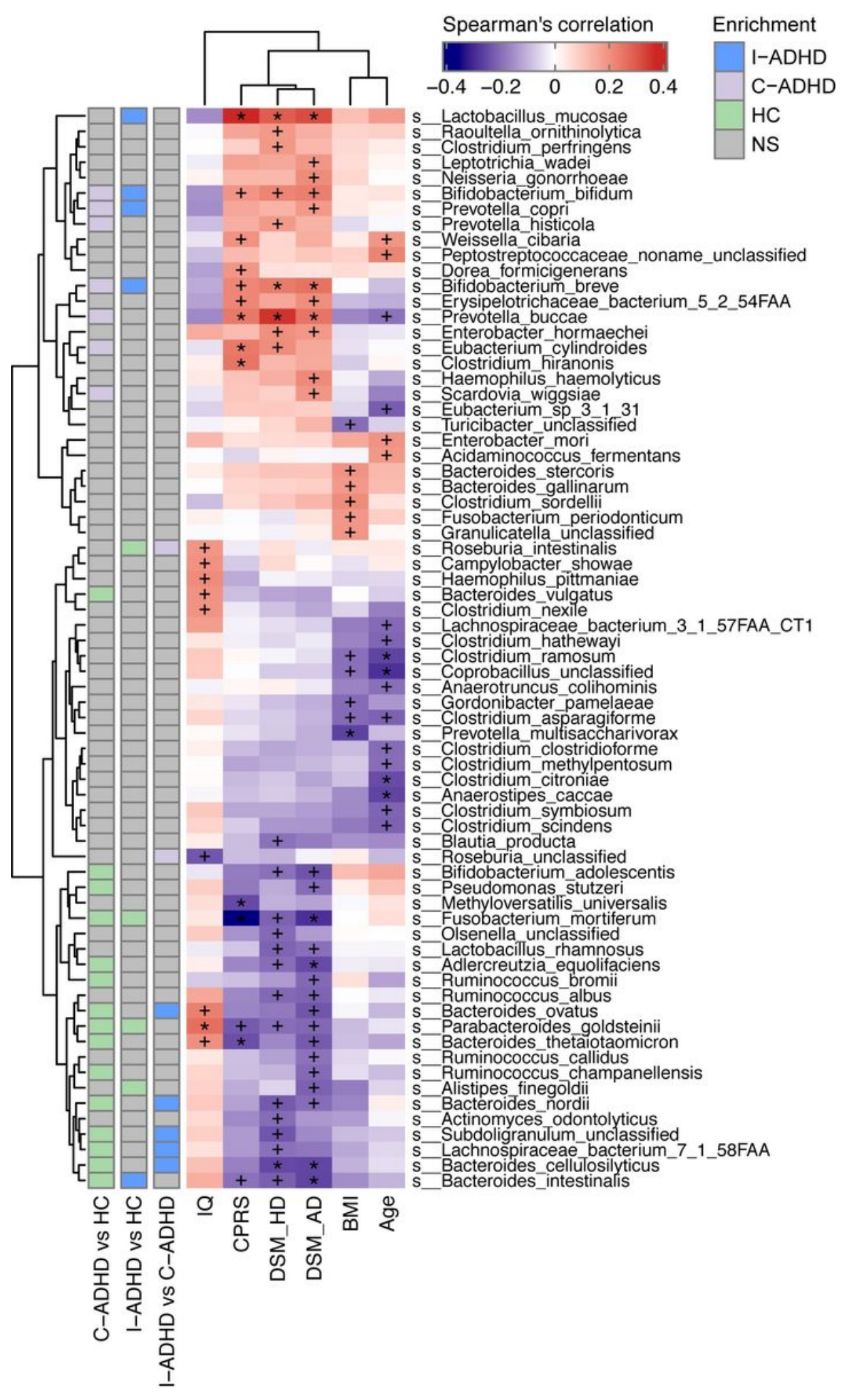

Figure 7

Correlation between gut microbiota species and ADHD clinical characteristics. Heat map of Spearman's correlation coefficient between the relative abundance of species and ADHD clinical characteristics (red and purple for positive and negative correlation, respectively). Species enrichment direction in different comparisons is shown on the left. Blue, I-ADHD-enriched; purple, C-ADHD-enriched; green, HC-enriched; gray, no significant difference. ' + ' denotes $p<0.01$; ‘ ${ }^{\prime}$ ' denotes $p<0.001$. 

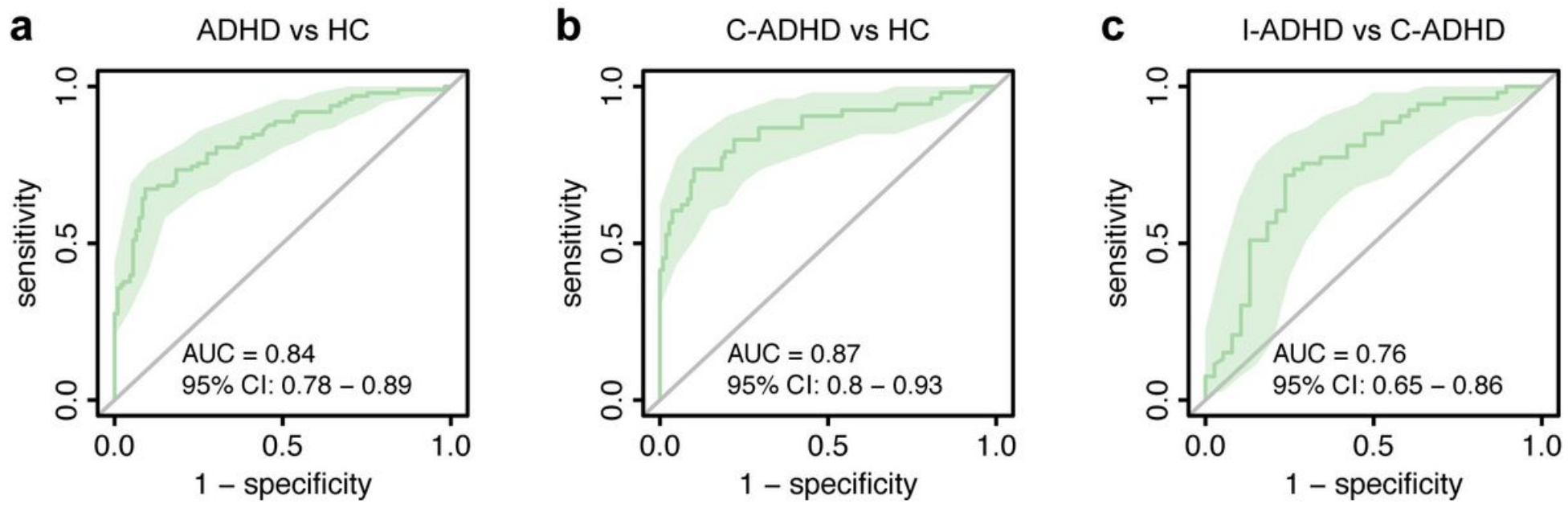

Figure 8

Classification of samples among different groups by relative abundance at the species level. (a) ROC generated between ADHD patients and HCs by 6 microbial markers selected by the random forest model. The AUC was 0.84 , and the $95 \% \mathrm{Cl}$ was $0.78-0.89$ (green area). (b) ROC generated between C-ADHD patients and HCs by 8 microbial markers selected from the random forest model. The AUC was 0.87 , and the $95 \% \mathrm{Cl}$ was $0.8-0.93$ (green area). (c) ROC generated between I-ADHD and C-ADHD patients by 35 microbial markers selected by the random forest model. The AUC was 0.76 , and the $95 \% \mathrm{Cl}$ was $0.65-0.86$ (green area).

\section{Supplementary Files}

This is a list of supplementary files associated with this preprint. Click to download.

- ADHDsupportinginformation120200514.docx

- ADHDsupportinginformation220200514.xlsx 